\title{
Synthesis and bioactivities of novel trifluoromethylated pyrazole oxime ether derivatives containing a pyridyl moiety
}

\author{
Hong Dai, Hai-Bo Yu, Jian-Bing Liu, Yong-Qiang Li, Xue Qin, Xin Zhang, Zhen-Fang Qin, \\ Ting-Ting Wang, and Jian-Xin Fang*
}

State Key Laboratory and Institute of Elemento-Organic Chemistry, Nankai University, Tianjin

300071, People's Republic of China

Email:nankaidx228@yahoo.com.cn

\begin{abstract}
A variety of novel 3-trifluoromethyl substituted pyrazole oxime ether derivatives containing a pyridyl moiety were designed and synthesized in satisfactory yields. Their structures were confirmed by ${ }^{1} \mathrm{H}$ NMR, ${ }^{13} \mathrm{C}$ NMR, and elemental analysis. The preliminary bioassays indicated that some of the title compounds showed potential insecticidal activities against Aphis craccivora, and some compounds also displayed plant growth regulatory activities.
\end{abstract}

Keywords: Pyrazole, oxime ether, trifluoromethyl, pyridyl, bioactivities

\section{Introduction}

The pyridyl ring is a prominent heterocyclic scaffold in lots of bioactive molecules. Numerous pyridine-based compounds have been reported to display versatile bioactivity, such as insecticidal, fungicidal, plant growth regulatory, anticancer, and antibacterial activity. ${ }^{1,2}$ Practical applications of pyridine derivatives in medicinal and pesticidal chemistry have also been achieved during the past two decades. For example, imidacloprid, acetamiprid, and thiacloprid (Figure 1), a class of neonicotinoid insecticides acting on the insect nicotinic acetylcholine receptors (nAChR), are currently used to control various insects such as aphids, whiteflies, and thrips on many crops due to their excellent insecticidal activities and good systemic properties. ${ }^{3}$ However, several aphid species have developed certain levels of neonicotinoids resistance after frequent field applications. ${ }^{4}$ Aphis craccivora belonging to pea aphids affects many crops of agricultural importance in Asia. To control Aphis craccivora more effectively, chemists devoted themselves to the development of neonicotinoids with new chemical structures and high insecticidal activities. ${ }^{5}$ 
On the other hand, substituted pyrazoles constitute an important class of compounds in the field of agricultural, and medicinal chemistry because of their broad spectrum biological activities. ${ }^{6}$ They are widely used as fungicide, ${ }^{7}$ insecticide, ${ }^{8}$ herbicide, ${ }^{9}$ and antitumor agent. ${ }^{10}$ As known, ethiprole and fipronil (Figure 1) are significant agricultural insecticides with extensive use for the effective control of the insects on corn and soybean, as well as stored grain insect pests. ${ }^{11}$

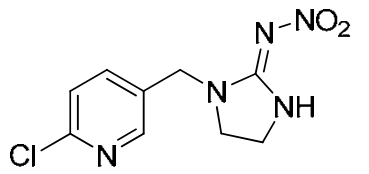

Imidacloprid

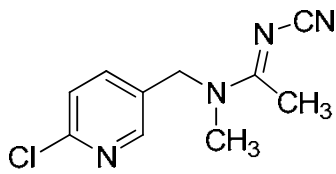

Acetamiprid

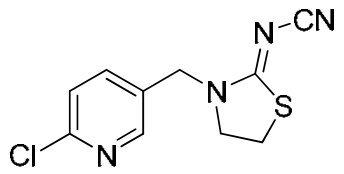

Thiacloprid

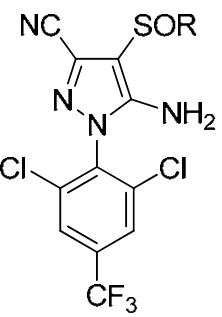

$\mathrm{R}=\mathrm{Et}$, Ethiprole

$\mathrm{R}=\mathrm{CF}_{3}$, Fipronil

Figure 1. Structures of imidacloprid, acetamiprid, thiacloprid, ethiprole and fipronil.

Recently, oxime ether derivatives have drawn much attention in medicinal research due to their significant bioactivity. ${ }^{12}$ Interestingly, Sun and co-workers found that some oxime ether compounds exhibited not only excellent insecticidal activities but have also good plant growth regulatory activities. ${ }^{13}$ In addition, many investigations have indicated that introducing a $\mathrm{CF}_{3}$ group into heterocyclic molecules mostly results in the improvement of physical, chemical and biological properties. ${ }^{14}$ Therefore, trifluoromethylated heterocycles became a focus of chemical research. Encouraged by these observations, we anticipated that introduction of the important pyridyl moiety and the $\mathrm{CF}_{3}$ group to pyrazole oxime ether molecules might generate a new group of biologically active compounds. Herein, we report the synthesis and bioactivity of some novel trifluoromethylated pyrazole oxime ether derivatives containing a pyridyl moiety.

\section{Results and Discussion}

\section{Chemistry}

The syntheses of the intermediates and target compounds were performed by the reactions illustrated in Schemes 1, 2, and 3. Intermediate 2-chloro-5-chloromethyl pyridine 4 was prepared from benzylamine as shown in Scheme 1. Benzylamine was condensed with propionaldehyde to afford compound $\mathbf{1}$ in $90 \%$ yield. Compound $\mathbf{1}$ was treated with triethylamine and acetic anhydride to produce compound $2{ }^{15}$ Then compound 2 reacted with a mixture of $\mathrm{POCl}_{3}$ and DMF

to form 2-chloro-5-methyl pyridine $3,{ }^{16}$ which was further converted to the intermediate 4 by the reaction with sulfuryl chloride in the presence of azodiisobutyronitrile. 


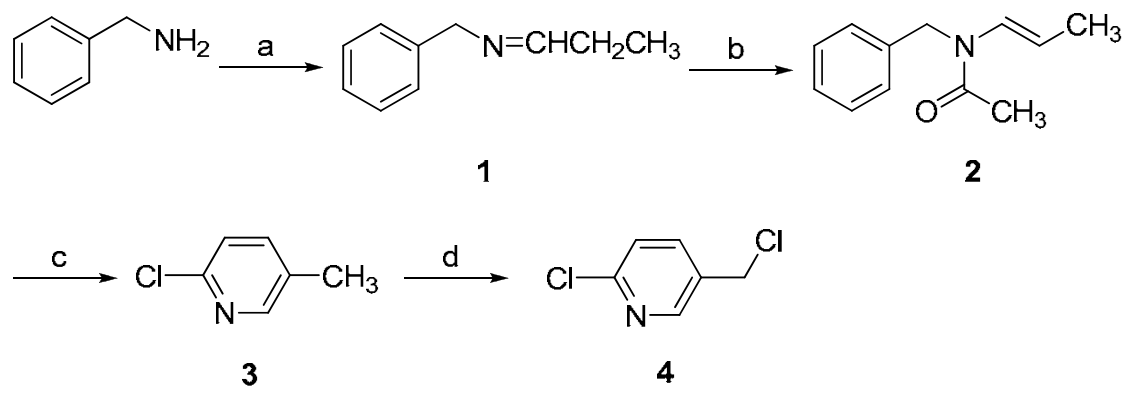

Scheme 1. Synthesis of the intermediate 4. (a) propionaldehyde, $\mathrm{KOH}, 5-25{ }^{\circ} \mathrm{C}, 4 \mathrm{~h}$; (b) $\left(\mathrm{CH}_{3} \mathrm{CO}\right)_{2} \mathrm{O}, \mathrm{Et}_{3} \mathrm{~N}, \mathrm{PhMe}, 5-20{ }^{\circ} \mathrm{C}, 3 \mathrm{~h}$; (c) $\mathrm{POCl}_{3}, \mathrm{DMF}, 100{ }^{\circ} \mathrm{C}, 17 \mathrm{~h}$; (d) $\mathrm{SO}_{2} \mathrm{Cl}_{2}$, azodiisobutyronitrile, benzene, $55^{\circ} \mathrm{C}$ for $1 \mathrm{~h}$ then reflux for $6 \mathrm{~h}$.

The condensation of ethyl 4,4,4-trifluoroacetoacetate with methylhydrazine in water afforded 5-hydroxypyrazole 5 (Scheme 2). Then, 5-hydroxypyrazole 5 was treated with $\mathrm{POCl}_{3}$ and $\mathrm{DMF}$ to give 4-formyl-5-chloropyrazole 6 in $87 \%$ yield. ${ }^{17}$ Subsequently, the substitution of 4-formyl-5-chloropyrazole 6 with phenols, benzenethiols, t-BuSH, and MeSNa under basic conditions, produced aldehydes 7a-7n (Scheme 2), 10a-10d (Scheme 3) in satisfactory yields. ${ }^{18}$ Aldehydes 7a-7n, 10a-10d were then converted to the corresponding oximes 8a-8n, 11a-11d by hydroxylamine under basic conditions, ${ }^{19}$ and further etherification of oximes 8a-8n, 11a-11d with the intermediate $\mathbf{4}$ under base promoting conditions generated the title compounds 9a-9n (Scheme 2), 12a-12d (Scheme 3). The best result for etherification $(\mathbf{8 a} \rightarrow \mathbf{9 a})$ was found to be $\mathrm{K}_{2} \mathrm{CO}_{3}$ in DMF at $90{ }^{\circ} \mathrm{C}$ (see Table 1). Consequently, $\mathrm{K}_{2} \mathrm{CO}_{3}$ and DMF were chosen as the best base and solvent, respectively to further synthesize other compounds $\mathbf{9 b}-\mathbf{9 n}, \mathbf{1 2 a - 1 2 d}$ in satisfactory yields (see Table 2).

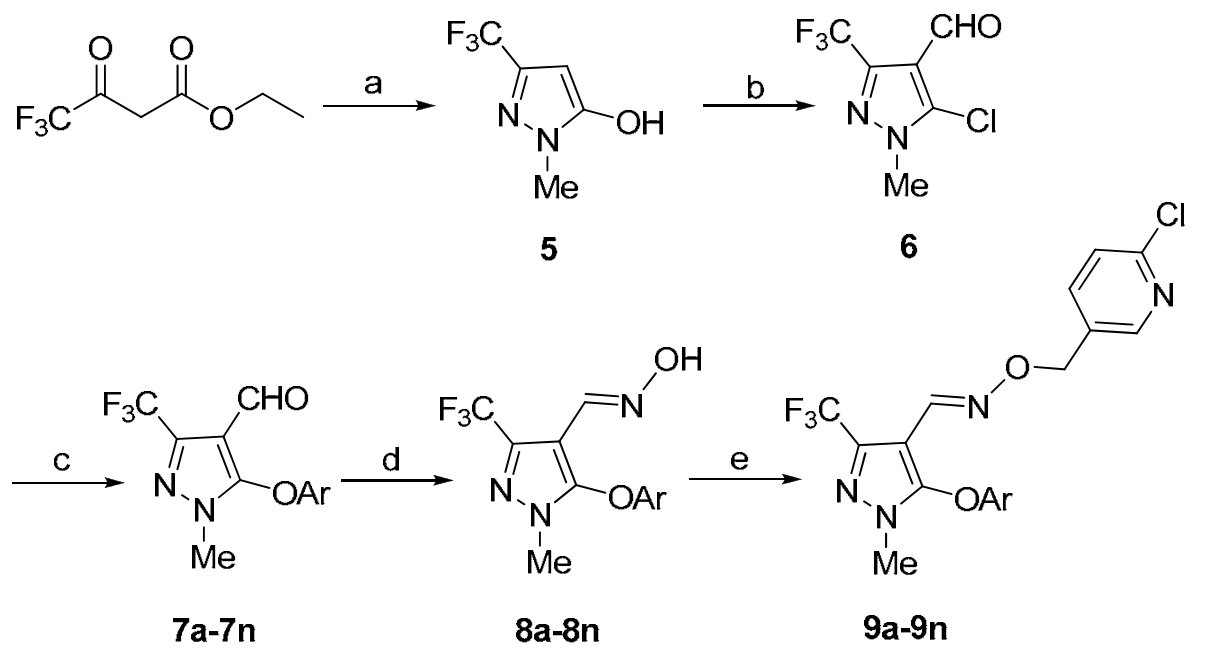

Scheme 2. Synthesis of compounds 9a-9n. (a) $\mathrm{CH}_{3} \mathrm{NHNH}_{2}, \mathrm{H}_{2} \mathrm{O}$, reflux, $12 \mathrm{~h}$; (b) $\mathrm{POCl}_{3}$, DMF, $55^{\circ} \mathrm{C}$ for $2 \mathrm{~h}$ then $10{ }^{\circ} \mathrm{C}$ for $5 \mathrm{~h}$; (c) $\mathrm{ArOH}, \mathrm{KOH}, \mathrm{Me}_{2} \mathrm{SO}, 100^{\circ} \mathrm{C}, 4-8 \mathrm{~h}$; (d) $\mathrm{NH}_{2} \mathrm{OH} \cdot \mathrm{HCl}, \mathrm{KOH}$, ethanol, reflux, 4-6 h; (e) 4, $\mathrm{K}_{2} \mathrm{CO}_{3}$, DMF, $90{ }^{\circ} \mathrm{C}, 10-14 \mathrm{~h}$. 


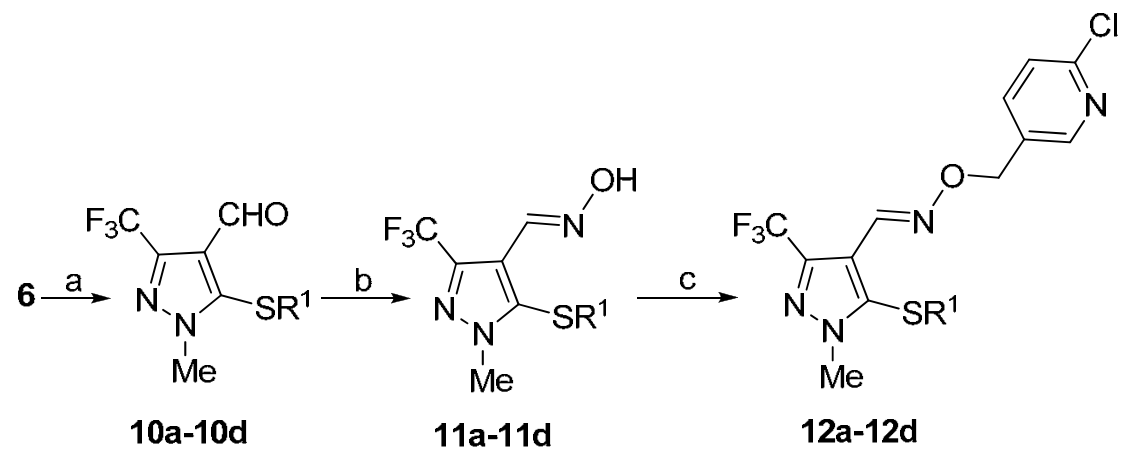

Scheme 3. Synthesis of compounds 12a-12d. (a) $\left(\mathrm{R}^{1}=\mathrm{Ar}, \mathrm{t}-\mathrm{Bu}\right)$ : Method A: $\mathrm{R}^{1} \mathrm{SH}, \mathrm{KOH}, \mathrm{DMF}$, $110^{\circ} \mathrm{C}, 6 \mathrm{~h} ;\left(\mathrm{R}^{1}=\mathrm{Me}\right)$ : Method B: $\mathrm{R}^{1} \mathrm{SNa}, \mathrm{DMF}, 70{ }^{\circ} \mathrm{C}, 3 \mathrm{~h}$; (b) $\mathrm{NH}_{2} \mathrm{OH} \cdot \mathrm{HCl}$, pyridine, n-butanol, reflux, 3-4 h; (c) 4, $\mathrm{K}_{2} \mathrm{CO}_{3}$, DMF, $90{ }^{\circ} \mathrm{C}$, 10-16 h.

Table 1. The effects of bases and solvents on the synthesis of compound 9a

\begin{tabular}{cccccc}
\hline Entry & $\begin{array}{c}\text { Temperature } \\
\left({ }^{\circ} \mathrm{C}\right)\end{array}$ & $\begin{array}{c}\text { Time } \\
(\mathrm{h})\end{array}$ & Base & Solvent & $\begin{array}{c}\text { Yield } \\
(\%)^{\mathrm{a}}\end{array}$ \\
\hline 1 & 90 & 12 & $\mathrm{Et} \mathrm{N}$ & $\mathrm{DMF}$ & 0 \\
2 & 90 & 12 & $\mathrm{NaOAc}$ & $\mathrm{DMF}$ & 22 \\
3 & 90 & 12 & $\mathrm{NaOEt}$ & $\mathrm{DMF}$ & 26 \\
4 & 90 & 12 & $\mathrm{NaH}$ & $\mathrm{DMF}$ & 49 \\
5 & 90 & 12 & $\mathrm{KOH}$ & $\mathrm{DMF}$ & 8 \\
6 & 90 & 12 & $\mathrm{~K}_{2} \mathrm{CO}_{3}$ & $\mathrm{DMF}$ & 88 \\
7 & 90 & 12 & $\mathrm{Pyridine}$ & $\mathrm{DMF}$ & 11 \\
8 & 90 & 12 & $\mathrm{~K}_{2} \mathrm{CO}_{3}$ & $\mathrm{Me} 2 \mathrm{SO}$ & 41 \\
9 & 90 & 12 & $\mathrm{~K}_{2} \mathrm{CO}_{3}$ & Dioxane & 38 \\
10 & 90 & 12 & $\mathrm{~K}_{2} \mathrm{CO}_{3}$ & $\mathrm{CH}_{3} \mathrm{CN}$ & 44 \\
11 & 90 & 12 & $\mathrm{~K}_{2} \mathrm{CO}_{3}$ & Butanone & 40 \\
12 & 90 & 12 & $\mathrm{~K}_{2} \mathrm{CO}_{3}$ & Toluene & 37 \\
\hline
\end{tabular}

${ }^{\mathrm{a}}$ Isolated yield.

Table 2. Reaction time and yields of 9a-9n, 12a-12d

\begin{tabular}{cccc}
\hline Compd & ArO or $\mathrm{R}^{1} \mathrm{~S}$ & Time $(\mathrm{h})$ & Yield (\%) \\
\hline 9a & $\mathrm{PhO}$ & 12 & 88 \\
9b & 2- $\mathrm{CH}_{3} \mathrm{PhO}$ & 14 & 86 \\
9c & 3- $\mathrm{CH}_{3} \mathrm{PhO}$ & 13 & 89 \\
9d & 4- $\mathrm{CH}_{3} \mathrm{PhO}$ & 10 & 93 \\
9e & 4-t-BuPhO & 12 & 88 \\
9f & 4- $\mathrm{CH}_{3} \mathrm{OPhO}$ & 10 & 93 \\
9g & $3,4-\left(\mathrm{CH}_{3}\right)_{2} \mathrm{PhO}$ & 11 & 88 \\
\hline
\end{tabular}


Table 2. Continued

\begin{tabular}{cccc}
\hline Compd & $\mathrm{ArO}$ or $\mathrm{R}^{1} \mathrm{~S}$ & Time $(\mathrm{h})$ & Yield $(\%)$ \\
\hline $\mathbf{9 h}$ & $2-\mathrm{ClPhO}$ & 13 & 87 \\
$\mathbf{9 i}$ & $3-\mathrm{ClPhO}$ & 13 & 89 \\
$\mathbf{9 j}$ & $4-\mathrm{ClPhO}$ & 11 & 92 \\
$\mathbf{9 k}$ & $4-\mathrm{FPhO}$ & 10 & 93 \\
$\mathbf{9 l}$ & $4-\mathrm{BrPhO}$ & 12 & 91 \\
$\mathbf{9 m}$ & $2,4-\mathrm{Cl}_{2} \mathrm{PhO}$ & 13 & 89 \\
$\mathbf{9 n}$ & $3-\mathrm{NO}_{2} \mathrm{PhO}$ & 14 & 87 \\
$\mathbf{1 2 a}$ & $3-\mathrm{CH}_{3} \mathrm{PhS}$ & 16 & 86 \\
$\mathbf{1 2 b}$ & $4-\mathrm{ClPhS}$ & 10 & 93 \\
$\mathbf{1 2 c}$ & t-BuS & 16 & 88 \\
$\mathbf{1 2 d}$ & $\mathrm{CH}_{3} \mathrm{~S}$ & 12 & 91 \\
\hline
\end{tabular}

All the title compounds were soluble in most organic solvents, which allowed a more easy use. Their structures were confirmed by ${ }^{1} \mathrm{H}$ NMR, ${ }^{13} \mathrm{C}$ NMR, and elemental analysis. In addition, the (E)-configuration of typical compound 12b was established on the basis of X-ray single-crystal structure analysis. ${ }^{20}$ In the asymmetric unit of compound 12b (Figure 2), there are two independent molecules. The bond lengths of $\mathrm{C}(12)-\mathrm{N}(3)(1.282$ (5) $\AA)$, and $\mathrm{C}(30)-\mathrm{N}(7)(1.282$ (5) $\AA)$ are approximate to that of normal $\mathrm{C}=\mathrm{N}(1.28 \AA)$ bond. ${ }^{21}$ The bond lengths of $\mathrm{C}(5)-\mathrm{N}(2)(1.457$ (5) $\AA$ ), and $\mathrm{C}(23)-\mathrm{N}(6)(1.442(5) \AA)$ are slightly shorter than that of normal single $\mathrm{C}-\mathrm{N}(1.47 \AA)$ bond. The torsion angles of $\mathrm{C}(3)-\mathrm{C}(12)-\mathrm{N}(3)-\mathrm{O}(1)$ and $\mathrm{C}(21)-\mathrm{C}(30)-\mathrm{N}(7)-\mathrm{O}(2)$ are $178.7(3)^{\circ}$ and $177.9(3)^{\circ}$, respectively, which indicates that the $\mathrm{C}=\mathrm{N}$ double bond is in the $(E)$-configuration.

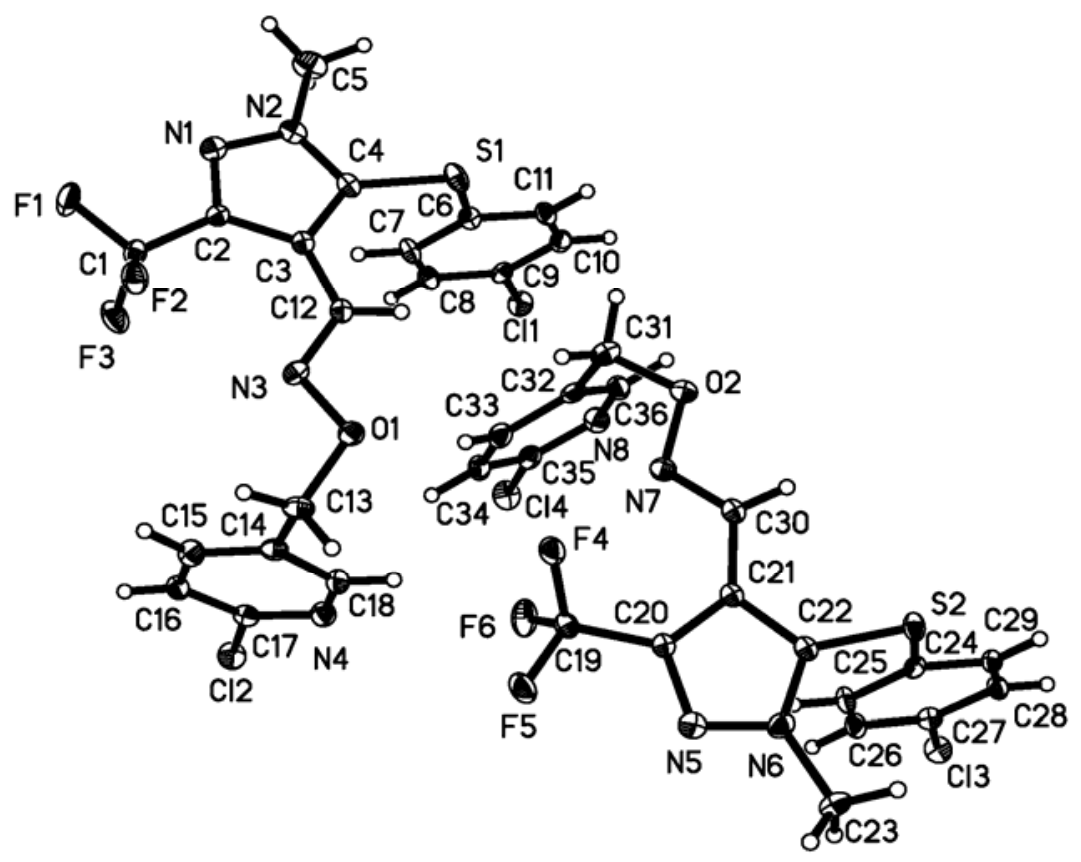

Figure 2. Molecular structure of compound 12b. 


\section{Biological activities}

The insecticidal activities of the target compounds 9a-9n, 12a-12d against pea aphids (Aphis craccivora) were investigated using a known procedure. ${ }^{22}$ As indicated in Table 3 , some of the title compounds showed good to excellent insecticidal activities against $A$. craccivora at the dosage of $0.5 \mathrm{mg} / \mathrm{mL}$. When the mortality of insects was recorded at $24 \mathrm{~h}$, compounds $\mathbf{9 b}, \mathbf{9 f}, \mathbf{9 g}$, 9j, 9k, 91, 9m, 9n, and 12b displayed excellent insecticidal activities against $A$. craccivora with the values of $92.2,90.1,90.4,91.3,90.2,91.5,90.8,90.1$, and $90.3 \%$, respectively, while the alkylthio derivatives 12c and 12d showed very low insecticidal activities against $A$. craccivora. When the mortality of insects was recorded at 48 h, compounds $\mathbf{9 a}, \mathbf{9 b}, \mathbf{9 f}, \mathbf{9 g}, \mathbf{9 j}, \mathbf{9 k}, \mathbf{9 1}, \mathbf{9 m}, \mathbf{9 n}$, and $\mathbf{1 2 b}$ exhibited around $90 \%$ inhibitory rate against $A$. craccivora. From the data listed in Table 3, we can find that among the chlorinated phenoxy derivatives, 4-substituted analogue $\mathbf{9 j}$ had more potency against $A$. craccivora than the corresponding 2- and 3-substituted analogues (9h and 9i); when the mortality of insects was recorded at 48 h, compounds $\mathbf{9 h}, \mathbf{9 i}$, and $\mathbf{9 j}$ displayed $72.4,75.7$, and $94.1 \%$ insecticidal activities against $A$. craccivora, respectively. At the same time, compound 12b containing a 4-chlorophenylthio moiety exhibited insecticidal activity similar to that of the phenoxy analogue $\mathbf{9 j}$. In addition, Table 4 showed the results of further toxicity assay about the typical candidates $\mathbf{9 f}, \mathbf{1 2 b}$, and the positive control nicotine sulfate against $A$. craccivora. The values of $\mathrm{LC}_{50}$ listed in Table 4 indicated that compounds $\mathbf{9 f}$ and $\mathbf{1 2 b}$ had slightly less potency against $A$. craccivora than the control nicotine sulfate. All the above results implied that introduction of the important pyridyl moiety and the $\mathrm{CF}_{3}$ group to pyrazole oxime ether molecules could afford some new compounds possessing potential insecticidal activities against $A$. craccivora. Further structural optimization and study of insecticidal activities are under way.

The plant growth regulatory activities of the title compounds $\mathbf{9 a - 9 n , ~ 1 2 a - 1 2 d ~ w e r e ~ e v a l u a t e d ~ b y ~}$ the cucumber cotyledon test at the concentration of $10 \mu \mathrm{g} / \mathrm{mL}$ according to a reported procedure. ${ }^{23}$ As shown in Table 3, some of the target compounds exhibited promising promotion effects on the radicle growth of cucumber cotyledons. Among these compounds, the promotional values of compounds 9a, 9e, 9f, 9j, 9k, 91, 9m, and 12b were 83.3, 83.6, 80.1, 98.3, 92.5, 98.0, 82.3, and $98.7 \%$, respectively. From the data listed in Table 3, we found that in the case of chlorinated phenoxy derivatives, the 4-substituted analogue $\mathbf{9 j}$ exhibited a much higher promotional activity than did the corresponding 2 - and 3 - substituted analogues (9h and $\mathbf{9 i}$ ). In addition, the phenylthio derivative 12b displayed promotional effect comparable to that of corresponding phenoxy analogue $\mathbf{9 j}$. It is interesting to note that the plant growth regulatory activities of compounds $\mathbf{9 j}$, 9k, 9l, and 12b are in accordance with their insecticidal activities to a certain extent.

In summary, a variety of novel 3-trifluoromethyl substituted pyrazole oxime ether derivatives containing a pyridyl moiety were synthesized in satisfactory yields. All the target compounds were soluble in most organic solvents, which allowed an easy use. The preliminary bioassays indicated that some of the designed compounds exhibited significant insecticidal activities against Aphis craccivora, and some compounds showed promising plant growth regulatory activities. 
Table 3. Insecticidal activities against $A$. craccivora and plant growth regulatory activities of the title compounds 9a-9n, 12a-12d

\begin{tabular}{cccc}
\hline \multirow{2}{*}{ Compd } & \multicolumn{2}{c}{$0.5 \mathrm{mg} / \mathrm{mL}(\text { mortality, \%) })^{\mathrm{a}}$} & \multirow{2}{*}{$\begin{array}{c}\text { rhizogenesis } \\
(10 \mu \mathrm{g} / \mathrm{mL}, \%)\end{array}$} \\
\cline { 2 - 3 } & $24 \mathrm{~h}$ & $48 \mathrm{~h}$ & $83.3 \pm 2.2$ \\
9a & $86.3 \pm 2.8$ & $88.6 \pm 2.3$ & $39.5 \pm 4.5$ \\
9b & $92.2 \pm 2.3$ & $94.1 \pm 2.6$ & $66.4 \pm 3.1$ \\
9c & $71.9 \pm 3.1$ & $74.3 \pm 3.5$ & $27.0 \pm 3.5$ \\
9d & $74.8 \pm 2.6$ & $78.4 \pm 2.3$ & $83.6 \pm 4.2$ \\
9e & $84.8 \pm 2.5$ & $87.9 \pm 2.6$ & $80.1 \pm 1.9$ \\
9f & $90.1 \pm 2.0$ & $92.3 \pm 3.2$ & $75.6 \pm 3.7$ \\
9g & $90.4 \pm 3.4$ & $92.3 \pm 2.4$ & $32.3 \pm 4.2$ \\
9h & $68.6 \pm 3.9$ & $72.4 \pm 3.8$ & $61.2 \pm 1.4$ \\
9i & $72.5 \pm 3.2$ & $75.7 \pm 2.2$ & $98.3 \pm 4.6$ \\
9j & $91.3 \pm 3.6$ & $94.1 \pm 4.6$ & $92.5 \pm 2.2$ \\
9k & $90.2 \pm 3.3$ & $92.1 \pm 2.3$ & $98.0 \pm 2.1$ \\
9l & $91.5 \pm 2.6$ & $94.4 \pm 2.0$ & $82.3 \pm 4.3$ \\
9m & $90.8 \pm 3.0$ & $93.5 \pm 1.6$ & $64.5 \pm 3.1$ \\
9n & $90.1 \pm 2.3$ & $91.2 \pm 3.2$ & $33.4 \pm 2.5$ \\
12a & $10.2 \pm 4.0$ & $11.2 \pm 2.4$ & $98.7 \pm 1.1$ \\
12b & $90.3 \pm 1.8$ & $92.3 \pm 2.1$ & $38.3 \pm 2.8$ \\
12c & $28.4 \pm 4.3$ & $32.2 \pm 3.1$ & $46.7 \pm 4.2$ \\
12d & $10.1 \pm 3.2$ & $10.9 \pm 3.6$ & \\
\hline
\end{tabular}

${ }^{\mathrm{a}}$ Each value represents the mean $\pm \mathrm{SD}$ of three experiments.

Table 4. Toxicities against Aphis craccivora of $\mathbf{9 f}, \mathbf{1 2 b}$, and Nicotine sulfate ${ }^{\mathrm{a}}$

\begin{tabular}{cccc}
\hline Compd & regression equation & $\mathrm{LC}_{50}{ }^{\mathrm{b}}(\mu \mathrm{g} / \mathrm{mL})$ & $r^{\mathrm{c}}$ \\
\hline $\mathbf{9 f}$ & $y=-1.41+3.07 x$ & 122.9 & 0.991 \\
$\mathbf{1 2 b}$ & $y=-0.90+2.77 x$ & 135.3 & 0.990 \\
$\mathbf{A}^{\mathrm{d}}$ & $y=0.96+2.12 x$ & 79.3 & 0.999 \\
\hline
\end{tabular}

${ }^{\mathrm{a}}$ The mortality was recorded at $48 \mathrm{~h} .{ }^{\mathrm{b}} \mathrm{LC}_{50}$ refers to median lethal concentration.

${ }^{\mathrm{c}} r$ refers to correlative coefficient. ${ }^{\mathrm{d}} \mathrm{A}$ refers to Nicotine sulfate. 


\section{Experimental Section}

General Procedures. Melting points were determined on an X-4 binocular microscope melting point apparatus (Beijing Tech Instrument Co., Beijing, China) and were uncorrected. ${ }^{1} \mathrm{H}$ NMR and ${ }^{13} \mathrm{C}$ NMR spectra were obtained on a Bruker AC-P 300 spectrometer $\left(300 \mathrm{MHz},{ }^{1} \mathrm{H} ; 75 \mathrm{MHz}\right.$, ${ }^{13} \mathrm{C}$ ) in $\mathrm{CDCl}_{3}$ with tetramethylsilane as the internal standard. Chemical shift values $(\delta)$ were given in ppm. Elemental analyses were determined on a Yanaco CHN Corder MT-3 elemental analyzer. All reagents were of analytical reagent grade or were chemically pure. All solvents were dried by standard methods and distilled prior to use.

Synthesis of 2-chloro-5-methylpyridine (3). ${ }^{16}$ To a stirred cold $\left(0{ }^{\circ} \mathrm{C}\right)$ solution of DMF $(54.6 \mathrm{~g}$, $0.75 \mathrm{~mol})$, was added dropwise phosphorus oxychloride $(22.3 \mathrm{~g}, 0.15 \mathrm{~mol})$. The resulting mixture was stirred at $10^{\circ} \mathrm{C}$ for $1 \mathrm{~h}$. To the above mixture, was added dropwise compound 2 (11.6 g, 0.06 mol), and then it was heated to $100{ }^{\circ} \mathrm{C}$ for $17 \mathrm{~h}$. After cooling to room temperature, the mixture was poured into water $(400 \mathrm{~mL})$, the mixture was continuously extracted with dichloromethane (4 $\times 100 \mathrm{~mL}$ ), and the organic layer was dried over anhydrous magnesium sulfate. The solvent was removed under reduced pressure to give 3 in $84 \%$ yield as a yellow oil. ${ }^{1} \mathrm{H}$ NMR (300 MHz, $\left.\mathrm{CDCl}_{3}\right): \delta 2.33\left(\mathrm{~s}, 3 \mathrm{H}, \mathrm{CH}_{3}\right), 7.20(\mathrm{~d}, J=8.1 \mathrm{~Hz}, 1 \mathrm{H}, \mathrm{Py}-\mathrm{H}), 7.48(\mathrm{~d}, J=7.8 \mathrm{~Hz}, 1 \mathrm{H}, \mathrm{Py}-\mathrm{H}), 8.25$ (s, 1H, Py-H).

Synthesis of 2-chloro-5-chloromethylpyridine (4). To a violently stirred warmed $\left(55^{\circ} \mathrm{C}\right)$ solution of 2-chloro-5-methylpyridine 3 (12.76 g, $0.10 \mathrm{~mol})$ and azodiisobutyronitrile $(0.03 \mathrm{~g})$ in benzene $(25 \mathrm{~mL})$, was added dropwise sulfuryl chloride $(9.45 \mathrm{~g}, 0.07 \mathrm{~mol})$. The resulting mixture was heated to reflux for $6 \mathrm{~h}$. The solvent was evaporated under reduced pressure, and the residue was subjected to column chromatography on silica gel (200-300 meshes) using a mixture of petroleum ether $\left(60-90{ }^{\circ} \mathrm{C}\right)$ and ethyl acetate as an eluent to obtain compound 4 in $67 \%$ yield as a colorless solid, mp 38-40 ${ }^{\circ} \mathrm{C} .{ }^{1} \mathrm{H}$ NMR (300 MHz, $\left.\mathrm{CDCl}_{3}\right): \delta 4.62\left(\mathrm{~s}, 2 \mathrm{H}, \mathrm{CH}_{2}\right), 7.30(\mathrm{~d}, J=8.4$ $\mathrm{Hz}, 1 \mathrm{H}, \mathrm{Py}-\mathrm{H}), 7.61$ (dd, $\left.J_{1}=8.1 \mathrm{~Hz}, J_{2}=2.1 \mathrm{~Hz}, 1 \mathrm{H}, \mathrm{Py}-\mathrm{H}\right), 8.31$ (d, $\left.J=2.4 \mathrm{~Hz}, 1 \mathrm{H}, \mathrm{Py}-\mathrm{H}\right)$.

Syntheses of intermediates 5 and 6. Intermediates 5 and $\mathbf{6}$ were synthesized according to literature procedures. ${ }^{17}$

1-Methyl-5-hydroxy-3-(trifluoromethyl)pyrazole (5). Yield, 85\%, white crystalline solid, mp $172-173{ }^{\circ} \mathrm{C} .{ }^{1} \mathrm{H}$ NMR $\left(300 \mathrm{MHz}, \mathrm{CDCl}_{3}\right): \delta 3.79$ (s, $\left.3 \mathrm{H}, \mathrm{N}^{-\mathrm{CH}_{3}}\right), 5.80(\mathrm{~s}, 1 \mathrm{H}, \mathrm{C}=\mathrm{CH})$.

5-Chloro-1-methyl-3-(trifluoromethyl)-4-pyrazolecarboxaldehyde (6). Yield, 87\%, yellow solid, mp 41-43 ${ }^{\circ} \mathrm{C} .{ }^{1} \mathrm{H}$ NMR (300 MHz, $\left.\mathrm{CDCl}_{3}\right): \delta 3.86$ (s, 3H, N-CH ), $9.86(\mathrm{~s}, 1 \mathrm{H}, \mathrm{CHO}$ ).

\section{General procedure for the preparation of aldehydes $(7 \mathbf{a}-7 \mathbf{n})^{18}$}

To a well stirred solution of substituted phenol $(6 \mathrm{mmol})$ in DMSO $(20 \mathrm{~mL})$, was added powdered potassium hydroxide $(7.5 \mathrm{mmol})$ in one portion at room temperature. The mixture was warmed to $55^{\circ} \mathrm{C}$ and stirred for $1 \mathrm{~h}$. To the above mixture, was added compound $6(5 \mathrm{mmol})$ in portions. Then, the solution was heated to $100{ }^{\circ} \mathrm{C}$ and maintained at this temperature for $4-8 \mathrm{~h}$. The cooled 
mixture was poured into ice-water $(80 \mathrm{~mL})$ and allowed to stand overnight. The precipitate was collected by filtration and washed with water $(3 \times 30 \mathrm{~mL})$ and hexane $(3 \times 30 \mathrm{~mL})$. The tan solid was dried under vacuum at $30{ }^{\circ} \mathrm{C}$ for $3 \mathrm{~h}$, yielding the corresponding aldehydes 7 , which were used without further purification except $\mathbf{7 b}$ and $\mathbf{7 g}$.

5-(2-Methylphenoxy)-3-(trifluoromethyl)-1-methyl-1H-Pyrazole-4-carbaldehyde (7b). Yield, 86\%, yellow solid, mp 67-69 ${ }^{\circ} \mathrm{C} .{ }^{1} \mathrm{H}$ NMR (300 MHz, $\left.\mathrm{CDCl}_{3}\right): \delta 2.42\left(\mathrm{~s}, 3 \mathrm{H}, \mathrm{Ar}^{-\mathrm{CH}_{3}}\right), 3.81$ (s, $\left.3 \mathrm{H}, \mathrm{N}-\mathrm{CH}_{3}\right), 6.62$ (d, $\left.J=8.7 \mathrm{~Hz}, 1 \mathrm{H}, \mathrm{Ar}-\mathrm{H}\right), 7.11-7.15$ (m, 2H, Ar-H), 7.29-7.32 (m, 1H, Ar-H), $9.55(\mathrm{~s}, 1 \mathrm{H}, \mathrm{CHO})$.

5-(3,4-Dimethylphenoxy)-3-(trifluoromethyl)-1-methyl-1H-Pyrazole-4-carbalde-hyde (7g). Yield, 83\%, yellow solid, mp 71-73 ${ }^{\circ} \mathrm{C} .{ }^{1} \mathrm{H}$ NMR (300 MHz, $\left.\mathrm{CDCl}_{3}\right): \delta 2.23\left(\mathrm{~s}, 3 \mathrm{H}, \mathrm{Ar}-\mathrm{CH}_{3}\right)$, $2.25\left(\mathrm{~s}, 3 \mathrm{H}, \mathrm{Ar}-\mathrm{CH}_{3}\right), 3.78\left(\mathrm{~s}, 3 \mathrm{H}, \mathrm{N}-\mathrm{CH}_{3}\right), 6.71\left(\mathrm{dd}, J_{1}=8.4 \mathrm{~Hz}, J_{2}=2.7 \mathrm{~Hz}, 1 \mathrm{H}, \mathrm{Ar}-\mathrm{H}\right), 6.79$ (d, $J=2.4 \mathrm{~Hz}, 1 \mathrm{H}, \mathrm{Ar}-\mathrm{H}), 7.10$ (d, $J=8.1 \mathrm{~Hz}, 1 \mathrm{H}, \mathrm{Ar}-\mathrm{H}), 9.60$ (s, 1H, CHO).

\section{General procedure for the preparation of aldehydes $(10 a-10 c)^{18}$}

To powdered potassium hydroxide $(6.5 \mathrm{mmol})$ was added water $(2 \mathrm{~mL})$, the mixture was stirred for $10 \mathrm{~min}$ at room temperature, and then a solution of substituted benzenethiol or t-BuSH (5.5 mmol) in DMF $(20 \mathrm{~mL})$ was added thereto. The resulting solution was heated to $55^{\circ} \mathrm{C}$ and stirred for $30 \mathrm{~min}$. Followed by addition of compound 6 ( $5 \mathrm{mmol})$, the reaction mixture was stirred at 110 ${ }^{\circ} \mathrm{C}$ for $6 \mathrm{~h}$, cooled, and poured into ice-water $(30 \mathrm{~mL})$. The precipitate was filtered to give a brown solid, which was washed with water and petroleum ether. The solid was dried under vacuum at 30 ${ }^{\circ} \mathrm{C}$ for $1 \mathrm{~h}$, affording the corresponding products, which were used without further purification except 10a.

5-(3-Methylphenylthio)-3-(trifluoromethyl)-1-methyl-1H-Pyrazole-4-carbaldehy-de (10a). Yield, 81\%, white solid, mp 79-81 ${ }^{\circ} \mathrm{C}$. ${ }^{1} \mathrm{H}$ NMR (300 MHz, $\left.\mathrm{CDCl}_{3}\right): \delta 2.32\left(\mathrm{~s}, 3 \mathrm{H}, \mathrm{Ar}-\mathrm{CH}_{3}\right), 3.89$ $\left(\mathrm{s}, 3 \mathrm{H}, \mathrm{N}-\mathrm{CH}_{3}\right), 6.97$ (d, J=7.5 Hz, 1H, Ar-H), 7.01 (s, 1H, Ar-H), 7.10 (d, J=7.5 Hz, 1H, Ar-H), 7.22 (t, $J=7.8 \mathrm{~Hz}, 1 \mathrm{H}, \mathrm{Ar}-\mathrm{H}), 10.07$ (s, 1H, CHO).

Synthesis of 5-Methylthio-3-(trifluoromethyl)-1-methyl-1H-Pyrazole-4-carbalde-hyde(10d). To a well stirred solution of $20 \%$ methylthio sodium $(25 \mathrm{mmol})$ in DMF $(40 \mathrm{~mL})$, was added compound 6 (12 mmol) in three portions, the reaction mixture was heated to $70{ }^{\circ} \mathrm{C}$ for $3 \mathrm{~h}$, cooled, and poured into ice-water $(100 \mathrm{~mL})$, the mixture was extracted with ethyl acetate $(4 \times 50 \mathrm{~mL})$ and the organic layer was dried over anhydrous magnesium sulfate. The solvent was removed under reduced pressure to afford aldehyde 10d. Yield, 78\%, yellow oil. ${ }^{1} \mathrm{H}$ NMR (300 $\left.\mathrm{MHz}, \mathrm{CDCl}_{3}\right): \delta$ $2.54\left(\mathrm{~s}, 3 \mathrm{H}, \mathrm{SCH}_{3}\right), 4.06\left(\mathrm{~s}, 3 \mathrm{H}, \mathrm{N}-\mathrm{CH}_{3}\right), 10.06$ (s, 1H, CHO).

\section{General procedure for the preparation of oximes $(8 a-8 n)^{19}$}

To a stirred solution of hydroxylamine hydrochloride $(18 \mathrm{mmol})$ in ethanol $(10 \mathrm{~mL})$, was added potassium hydroxide $(20 \mathrm{mmol})$. The resulting mixture was stirred at room temperature for 20 min, then a solution of aldehydes 7a-7n $(15 \mathrm{mmol})$ in ethanol $(15 \mathrm{~mL})$ was added dropwise. The mixture was then heated to reflux for $4-6 \mathrm{~h}$ and cooled to room temperature. The reaction mixture 
was poured into water $(100 \mathrm{~mL})$ and extracted with dichloromethane $(4 \times 40 \mathrm{~mL})$. The organic layer was dried over anhydrous sodium sulfate. The solvent was condensed to give pyrazole oximes 8, which were used directly without further purification except $\mathbf{8 b}$ and $\mathbf{8 g}$.

5-(2-Methylphenoxy)-3-(trifluoromethyl)-1-methyl-1H-Pyrazole-4-carbaldehyde-oxime (8b). Yield 85\%, yellow solid, mp 120-122 ${ }^{\circ} \mathrm{C} .{ }^{1} \mathrm{H}$ NMR $\left(300 \mathrm{MHz}, \mathrm{CDCl}_{3}\right): \delta 2.39\left(\mathrm{~s}, 3 \mathrm{H}, \mathrm{Ar}^{-\mathrm{CH}_{3}}\right)$, $3.67\left(\mathrm{~s}, 3 \mathrm{H}, \mathrm{N}-\mathrm{CH}_{3}\right), 6.47$ (d, $\left.J=8.4 \mathrm{~Hz}, 1 \mathrm{H}, \mathrm{Ar}-\mathrm{H}\right), 7.01-7.10(\mathrm{~m}, 2 \mathrm{H}, \mathrm{Ar}-\mathrm{H}), 7.25$ (d, J = 7.5 Hz, $1 \mathrm{H}, \mathrm{Ar}-\mathrm{H}), 7.88(\mathrm{~s}, 1 \mathrm{H}, \mathrm{CH}=\mathrm{N}), 8.24(\mathrm{~s}, 1 \mathrm{H}, \mathrm{N}-\mathrm{OH})$.

5-(3,4-Dimethylphenoxy)-3-(trifluoromethyl)-1-methyl-1H-Pyrazole-4-carbalde-hydeoxime (8g). Yield 89\%, yellow solid, mp 130-132 ${ }^{\circ} \mathrm{C} .{ }^{1} \mathrm{H}$ NMR $\left(300 \mathrm{MHz}, \mathrm{CDCl}_{3}\right): \delta 2.23(\mathrm{~s}, 6 \mathrm{H}$, $\left.2 \times \mathrm{Ar}^{-\mathrm{CH}_{3}}\right), 3.68\left(\mathrm{~s}, 3 \mathrm{H}, \mathrm{N}-\mathrm{CH}_{3}\right), 6.58(\mathrm{~d}, J=8.4 \mathrm{~Hz}, 1 \mathrm{H}, \mathrm{Ar}-\mathrm{H}), 6.67(\mathrm{~s}, 1 \mathrm{H}, \mathrm{Ar}-\mathrm{H}), 7.05(\mathrm{~d}, J=$ $8.4 \mathrm{~Hz}, 1 \mathrm{H}, \mathrm{Ar}-\mathrm{H}), 7.94(\mathrm{~s}, 1 \mathrm{H}, \mathrm{CH}=\mathrm{N}), 8.06$ (s, 1H, N-OH).

\section{General procedure for the preparation of oximes (11a-11d) ${ }^{19}$}

To a stirred solution of aldehydes 10a-10d $(5 \mathrm{mmol})$ and hydroxylamine hydrochloride $(10 \mathrm{mmol})$ in n-butanol $(20 \mathrm{~mL})$, a solution of pyridine $(15 \mathrm{mmol})$ in n-butanol $(10 \mathrm{~mL})$ was added dropwise at room temperature. The reaction mixture was stirred at room temperature for $30 \mathrm{~min}$, and then heated to reflux for 3-4 h. After the solvent was evaporated under reduced pressure, the residue was admixed with water $(60 \mathrm{~mL})$, and extracted with diethyl ether $(3 \times 50 \mathrm{~mL})$. The combined organic layer was washed with $10 \% \mathrm{NaHCO}_{3}$ solution $(3 \times 20 \mathrm{~mL})$, dried over anhydrous magnesium sulfate and concentrated to produce pyrazole oximes 11, which were used for the next reaction without further purification except 11a and 11d.

5-(3-Methylphenylthio)-3-(trifluoromethyl)-1-methyl-1H-Pyrazole-4-carbaldehy-deoxime (11a). Yield 84\%, white solid, mp 118-120 ${ }^{\circ} \mathrm{C} .{ }^{1} \mathrm{H}$ NMR $\left(300 \mathrm{MHz}, \mathrm{CDCl}_{3}\right): \delta 2.30(\mathrm{~s}, 3 \mathrm{H}$, Ar- $\left.\mathrm{CH}_{3}\right), 3.87$ (s, 3H, N-CH 3$), 6.84$ (d, J=7.8 Hz, 1H, Ar-H), 6.90 (s, 1H, Ar-H), 7.04 (d, J= 7.5 $\mathrm{Hz}, 1 \mathrm{H}, \mathrm{Ar}-\mathrm{H}), 7.17$ (t, J=7.5 Hz, 1H, Ar-H), $8.24(\mathrm{~s}, 1 \mathrm{H}, \mathrm{CH}=\mathrm{N}), 8.72(\mathrm{~s}, 1 \mathrm{H}, \mathrm{N}-\mathrm{OH})$.

5-Methylthio-3-(trifluoromethyl)-1-methyl-1H-Pyrazole-4-carbaldehydeoxime (11d). Yield $89 \%$, white solid, mp 133-135 ${ }^{\circ} \mathrm{C} .{ }^{1} \mathrm{H}$ NMR $\left(300 \mathrm{MHz}, \mathrm{CDCl}_{3}\right): \delta 2.38\left(\mathrm{~s}, 3 \mathrm{H}, \mathrm{SCH}_{3}\right), 4.05(\mathrm{~s}, 3 \mathrm{H}$, $\left.\mathrm{N}-\mathrm{CH}_{3}\right), 8.26(\mathrm{~s}, 1 \mathrm{H}, \mathrm{CH}=\mathrm{N}), 9.10(\mathrm{~s}, 1 \mathrm{H}, \mathrm{N}-\mathrm{OH})$.

General procedure for the preparation of the title compounds (9a-9n, 12a-12d)

To a mixture of 2-chloro-5-chloromethylpyridine 4 ( $5 \mathrm{mmol})$, oximes 8a-8n or 11a-11d (4 mmol), and DMF $(30 \mathrm{~mL})$, potassium carbonate $(10 \mathrm{mmol})$ was added thereto. The reaction mixture was heated gradually to $90{ }^{\circ} \mathrm{C}$ and stirred for $10-16 \mathrm{~h}$. The resulting mixture was cooled, diluted with ice water $(150 \mathrm{~mL})$, and extracted with dichloromethane $(4 \times 50 \mathrm{~mL})$. The organic layer was washed with saturated brine $(2 \times 20 \mathrm{~mL})$, and then dried over anhydrous sodium sulfate. The solvent was removed under reduced pressure, and the crude residue was purified by column chromatography on silica gel with the solvent system of ethyl acetate and petroleum ether (60-90 ${ }^{\circ} \mathrm{C}$ ) to afford the target compounds 9a-9n, 12a-12d.

5-(Phenoxy)-3-(trifluoromethyl)-1-methyl-1H-Pyrazole-4-carbaldehyde-O-((2-chloropyridi n-5-yl)methyl)oxime (9a). Yield 88\%, yellow solid, mp 55-57 ${ }^{\circ} \mathrm{C}$. ${ }^{1} \mathrm{H} \mathrm{NMR}\left(300 \mathrm{MHz}, \mathrm{CDCl}_{3}\right)$ : 
$\delta 3.75$ (s, 3H, N-CH $), 4.82\left(\mathrm{~s}, 2 \mathrm{H}, \mathrm{CH}_{2}\right), 6.83$ (d, J=8.4 Hz, 2H, Ar-H), 7.12-7.18 (m, 2H, Py-H and $\mathrm{Ar}-\mathrm{H}), 7.30(\mathrm{~d}, J=8.1 \mathrm{~Hz}, 2 \mathrm{H}, \mathrm{Ar}-\mathrm{H}), 7.36\left(\mathrm{dd}, J_{1}=8.1 \mathrm{~Hz}, J_{2}=2.4 \mathrm{~Hz}, 1 \mathrm{H}, \mathrm{Py}-\mathrm{H}\right), 7.92(\mathrm{~s}$, $1 \mathrm{H}, \mathrm{CH}=\mathrm{N}), 8.15(\mathrm{~d}, J=2.1 \mathrm{~Hz}, 1 \mathrm{H}, \mathrm{Py}-\mathrm{H}) .{ }^{13} \mathrm{C} \mathrm{NMR}\left(75 \mathrm{MHz}, \mathrm{CDCl}_{3}\right): \delta 35.2,72.6,100.7$, 115.1, 118.9, 122.5, 123.9, 124.0, 129.9, 132.0, 138.8, 138.9, 147.3, 149.5, 150.9, 155.7. Anal. Calcd for $\mathrm{C}_{18} \mathrm{H}_{14} \mathrm{ClF}_{3} \mathrm{~N}_{4} \mathrm{O}_{2}$ : C, 52.63; H, 3.44; N, 13.64. Found: C, 52.68; H, 3.35; N, 13.58 .

5-(2-Methylphenoxy)-3-(trifluoromethyl)-1-methyl-1H-Pyrazole-4-carbaldehyde-O-((2-chlo ropyridin-5-yl)methyl)oxime (9b). Yield $86 \%$, yellow solid, mp 56-58 ${ }^{\circ} \mathrm{C} .{ }^{1} \mathrm{H} \mathrm{NMR}(300 \mathrm{MHz}$, $\left.\mathrm{CDCl}_{3}\right): \delta 2.33\left(\mathrm{~s}, 3 \mathrm{H}, \mathrm{Ar}-\mathrm{CH}_{3}\right), 3.75\left(\mathrm{~s}, 3 \mathrm{H}, \mathrm{N}-\mathrm{CH}_{3}\right), 4.81\left(\mathrm{~s}, 2 \mathrm{H}, \mathrm{CH}_{2}\right), 6.43$ (t, J= $7.5 \mathrm{~Hz}, 1 \mathrm{H}$, Ar-H), 7.03-7.07 (m, 2H, Ar-H), 7.15 (d, $J=8.1 \mathrm{~Hz}, 1 \mathrm{H}, \mathrm{Py}-\mathrm{H}), 7.20-7.23$ (m, 1H, Ar-H), 7.31 $\left(\mathrm{dd}, J_{1}=8.1 \mathrm{~Hz}, J_{2}=2.4 \mathrm{~Hz}, 1 \mathrm{H}, \mathrm{Py}-\mathrm{H}\right), 7.89(\mathrm{~s}, 1 \mathrm{H}, \mathrm{CH}=\mathrm{N}), 8.13(\mathrm{~d}, J=2.4 \mathrm{~Hz}, 1 \mathrm{H}, \mathrm{Py}-\mathrm{H}) .{ }^{13} \mathrm{C}$ NMR (75 MHz, $\left.\mathrm{CDCl}_{3}\right): \delta 16.1,35.1,72.5,100.2,112.8,115.4,118.9,122.5,123.9,126.7,127.1$, 131.6, 132.1, 138.7, 138.9, 147.8, 149.2, 150.8, 154.0. Anal. Calcd for $\mathrm{C}_{19} \mathrm{H}_{16} \mathrm{ClF}_{3} \mathrm{~N}_{4} \mathrm{O}_{2}$ : C, 53.72; H, 3.80; N, 13.19. Found: C, 53.86; H, 3.95; N, 13.10 .

5-(3-Methylphenoxy)-3-(trifluoromethyl)-1-methyl-1H-Pyrazole-4-carbaldehyde-O-((2-chlo ropyridin-5-yl)methyl)oxime (9c). Yield 89\%, yellow solid, mp 50-52 ${ }^{\circ} \mathrm{C} .{ }^{1} \mathrm{H}$ NMR $(300 \mathrm{MHz}$, $\left.\mathrm{CDCl}_{3}\right): \delta 2.32\left(\mathrm{~s}, 3 \mathrm{H}, \mathrm{Ar}-\mathrm{CH}_{3}\right), 3.73\left(\mathrm{~s}, 3 \mathrm{H}, \mathrm{N}-\mathrm{CH}_{3}\right), 4.86\left(\mathrm{~s}, 2 \mathrm{H}, \mathrm{CH}_{2}\right), 6.63(\mathrm{~s}, 1 \mathrm{H}, \mathrm{Ar}-\mathrm{H}), 6.72$ $(\mathrm{d}, J=8.7 \mathrm{~Hz}, 1 \mathrm{H}, \mathrm{Ar}-\mathrm{H}), 6.94-7.22\left(\mathrm{~m}, 3 \mathrm{H}, \mathrm{Py}-\mathrm{H}\right.$ and Ar-H), $7.36\left(\mathrm{dd}, J_{1}=8.1 \mathrm{~Hz}, J_{2}=2.1 \mathrm{~Hz}\right.$, $1 \mathrm{H}, \mathrm{Py}-\mathrm{H}), 7.92(\mathrm{~s}, 1 \mathrm{H}, \mathrm{CH}=\mathrm{N}), 8.16(\mathrm{~d}, J=2.4 \mathrm{~Hz}, 1 \mathrm{H}, \mathrm{Py}-\mathrm{H}) .{ }^{13} \mathrm{C}$ NMR $\left(75 \mathrm{MHz}, \mathrm{CDCl}_{3}\right): \delta$ 21.4, 35.2, 72.6, 100.8, 112.1, 115.6, 119.0, 122.5, 123.9, 124.8, 129.7, 130.4, 132.0, 133.6, 138.8, 140.4, 149.5, 150.9, 153.7. Anal. Calcd for $\mathrm{C}_{19} \mathrm{H}_{16} \mathrm{ClF}_{3} \mathrm{~N}_{4} \mathrm{O}_{2}: \mathrm{C}, 53.72 ; \mathrm{H}, 3.80 ; \mathrm{N}, 13.19$. Found: C, 53.66; H, 3.72; N, 13.30 .

5-(4-Methylphenoxy)-3-(trifluoromethyl)-1-methyl-1H-Pyrazole-4-carbaldehyde-O-((2-chlo ropyridin-5-yl)methyl)oxime (9d). Yield 93\%, yellow solid, mp 73-75 ${ }^{\circ} \mathrm{C} .{ }^{1} \mathrm{H} \mathrm{NMR}(300 \mathrm{MHz}$, $\left.\mathrm{CDCl}_{3}\right): \delta 2.34\left(\mathrm{~s}, 3 \mathrm{H}, \mathrm{Ar}-\mathrm{CH}_{3}\right), 3.72\left(\mathrm{~s}, 3 \mathrm{H}, \mathrm{N}-\mathrm{CH}_{3}\right), 4.85\left(\mathrm{~s}, 2 \mathrm{H}, \mathrm{CH}_{2}\right), 6.71(\mathrm{~d}, J=8.4 \mathrm{~Hz}, 2 \mathrm{H}$, Ar-H), 7.09 (d, $J=8.4 \mathrm{~Hz}, 2 \mathrm{H}, \mathrm{Ar}-\mathrm{H}), 7.15$ (d, $J=8.4 \mathrm{~Hz}, 1 \mathrm{H}, \mathrm{Py}-\mathrm{H}), 7.38\left(\mathrm{dd}, J_{1}=8.4 \mathrm{~Hz}, J_{2}=\right.$ $2.4 \mathrm{~Hz}, 1 \mathrm{H}, \mathrm{Py}-\mathrm{H}), 7.90(\mathrm{~s}, 1 \mathrm{H}, \mathrm{CH}=\mathrm{N}), 8.17$ (d, $J=2.1 \mathrm{~Hz}, 1 \mathrm{H}, \mathrm{Py}-\mathrm{H}) .{ }^{13} \mathrm{C} \mathrm{NMR}(75 \mathrm{MHz}$, $\left.\mathrm{CDCl}_{3}\right): \delta 20.6,35.2,72.6,100.6,114.9,119.0,122.5,123.8,130.4,132.0,133.6,138.8,139.0$, 147.7, 149.5, 150.8, 153.8. Anal. Calcd for $\mathrm{C}_{19} \mathrm{H}_{16} \mathrm{ClF}_{3} \mathrm{~N}_{4} \mathrm{O}_{2}$ : C, 53.72; H, 3.80; N, 13.19. Found: C, 53.74; H, 3.65; N, 13.25 .

5-(4-t-Butylphenoxy)-3-(trifluoromethyl)-1-methyl-1H-Pyrazole-4-carbaldehyde-O-((2-chlo ropyridin-5-yl)methyl)oxime (9e). Yield $88 \%$, yellow solid, mp $45-47{ }^{\circ} \mathrm{C} .{ }^{1} \mathrm{H}$ NMR $(300 \mathrm{MHz}$, $\left.\mathrm{CDCl}_{3}\right): \delta 1.31\left(\mathrm{~s}, 9 \mathrm{H}, \mathrm{C}\left(\mathrm{CH}_{3}\right)_{3}\right), 3.73\left(\mathrm{~s}, 3 \mathrm{H}, \mathrm{N}-\mathrm{CH}_{3}\right), 4.82\left(\mathrm{~s}, 2 \mathrm{H}, \mathrm{CH}_{2}\right), 6.77(\mathrm{~d}, J=8.4 \mathrm{~Hz}, 2 \mathrm{H}$, Ar-H), 7.18 (d, $J=8.1 \mathrm{~Hz}, 1 \mathrm{H}, \mathrm{Py}-\mathrm{H}), 7.34$ (d, $J=8.4 \mathrm{~Hz}, 2 \mathrm{H}, \mathrm{Ar}-\mathrm{H}), 7.45$ (dd, $J_{1}=8.1 \mathrm{~Hz}, J_{2}=$ $2.1 \mathrm{~Hz}, 1 \mathrm{H}, \mathrm{Py}-\mathrm{H}), 7.88$ (s, $1 \mathrm{H}, \mathrm{CH}=\mathrm{N}), 8.19$ (d, $J=2.1 \mathrm{~Hz}, 1 \mathrm{H}, \mathrm{Py}-\mathrm{H}) .{ }^{13} \mathrm{C} \mathrm{NMR}(75 \mathrm{MHz}$, $\left.\mathrm{CDCl}_{3}\right): \delta 31.4,34.4,35.3,67.6,100.5,114.7,118.9,122.5,126.5,126.8,136.5,139.2,140.5$, 147.1, 147.9, 152.8, 153.7. Anal. Calcd for $\mathrm{C}_{22} \mathrm{H}_{22} \mathrm{ClF}_{3} \mathrm{~N}_{4} \mathrm{O}_{2}$ : C, 56.60; H, 4.75; N, 12.00. Found: C, 56.65; H, 4.84; N, 11.87 .

5-(4-Methoxyphenoxy)-3-(trifluoromethyl)-1-methyl-1H-Pyrazole-4-carbaldehy-de-O-((2-c hloropyridin-5-yl)methyl)oxime (9f). Yield 93\%, white solid, mp 93-95 ${ }^{\circ} \mathrm{C} .{ }^{1} \mathrm{H} \mathrm{NMR}(300 \mathrm{MHz}$, $\left.\mathrm{CDCl}_{3}\right): \delta 3.74\left(\mathrm{~s}, 3 \mathrm{H}, \mathrm{N}-\mathrm{CH}_{3}\right), 3.80\left(\mathrm{~s}, 3 \mathrm{H}, \mathrm{Ar}-\mathrm{OCH}_{3}\right), 4.88\left(\mathrm{~s}, 2 \mathrm{H}, \mathrm{CH}_{2}\right), 6.77(\mathrm{~d}, J=9.3 \mathrm{~Hz}, 2 \mathrm{H}$, 
Ar-H), $6.82(\mathrm{~d}, J=9.3 \mathrm{~Hz}, 2 \mathrm{H}, \mathrm{Ar}-\mathrm{H}), 7.19(\mathrm{~d}, J=8.1 \mathrm{~Hz}, 1 \mathrm{H}, \mathrm{Py}-\mathrm{H}), 7.40\left(\mathrm{dd}, J_{1}=8.1 \mathrm{~Hz}, J_{2}=\right.$ $2.1 \mathrm{~Hz}, 1 \mathrm{H}, \mathrm{Py}-\mathrm{H}), 7.90(\mathrm{~s}, 1 \mathrm{H}, \mathrm{CH}=\mathrm{N}), 8.18(\mathrm{~d}, J=2.1 \mathrm{~Hz}, 1 \mathrm{H}, \mathrm{Py}-\mathrm{H}) .{ }^{13} \mathrm{C}$ NMR $(75 \mathrm{MHz}$, $\left.\mathrm{CDCl}_{3}\right): \delta 35.2,55.7,72.6,100.4,114.9,116.1,118.9,122.5,123.9,132.0,138.8,138.9,148.0$, 149.5, 149.7, 150.9, 156.0. Anal. Calcd for $\mathrm{C}_{19} \mathrm{H}_{16} \mathrm{ClF}_{3} \mathrm{~N}_{4} \mathrm{O}_{3}: \mathrm{C}, 51.77 ; \mathrm{H}, 3.66 ; \mathrm{N}, 12.71$. Found: C, 51.82; H, 3.55; N, 12.83 .

5-(3,4-Dimethylphenoxy)-3-(trifluoromethyl)-1-methyl-1H-Pyrazole-4-carbalde-hyde-O-((2 -chloropyridin-5-yl)methyl)oxime (9g). Yield 88\%, yellow solid, mp 67-69 ${ }^{\circ} \mathrm{C}$. ${ }^{1} \mathrm{H}$ NMR (300 $\left.\mathrm{MHz}, \mathrm{CDCl}_{3}\right): \delta 2.22\left(\mathrm{~s}, 3 \mathrm{H}, \mathrm{Ar}-\mathrm{CH}_{3}\right), 2.24\left(\mathrm{~s}, 3 \mathrm{H}, \mathrm{Ar}-\mathrm{CH}_{3}\right), 3.72\left(\mathrm{~s}, 3 \mathrm{H}, \mathrm{N}-\mathrm{CH}_{3}\right), 4.88(\mathrm{~s}, 2 \mathrm{H}$, $\left.\mathrm{CH}_{2}\right), 6.55\left(\mathrm{dd}, J_{1}=8.1 \mathrm{~Hz}, J_{2}=2.7 \mathrm{~Hz}, 1 \mathrm{H}, \mathrm{Ar}-\mathrm{H}\right), 6.61(\mathrm{~d}, J=2.4 \mathrm{~Hz}, 1 \mathrm{H}, \mathrm{Ar}-\mathrm{H}), 7.04(\mathrm{~d}, J=$ $8.4 \mathrm{~Hz}, 1 \mathrm{H}, \mathrm{Ar}-\mathrm{H}), 7.14$ (d, $J=8.4 \mathrm{~Hz}, 1 \mathrm{H}, \mathrm{Py}-\mathrm{H}), 7.39$ (dd, $\left.J_{1}=8.1 \mathrm{~Hz}, J_{2}=2.4 \mathrm{~Hz}, 1 \mathrm{H}, \mathrm{Py}-\mathrm{H}\right)$, $7.90(\mathrm{~s}, 1 \mathrm{H}, \mathrm{CH}=\mathrm{N}), 8.17(\mathrm{~d}, J=2.1 \mathrm{~Hz}, 1 \mathrm{H}, \mathrm{Py}-\mathrm{H}) .{ }^{13} \mathrm{C} \mathrm{NMR}\left(75 \mathrm{MHz}, \mathrm{CDCl}_{3}\right): \delta 18.9,20.0$, 35.2 , 72.6, 100.7, 112.2, 116.2, 118.9, 122.5, 123.8, 130.7, 132.0, 132.3, 138.6, 138.9, 139.0, 147.8, 149.6, 150.8, 153.9. Anal. Calcd for $\mathrm{C}_{20} \mathrm{H}_{18} \mathrm{ClF}_{3} \mathrm{~N}_{4} \mathrm{O}_{2}$ : C, 54.74; $\mathrm{H}, 4.13 ; \mathrm{N}, 12.77$. Found: C, 54.86; H, 4.28; N, 12.62 .

5-(2-Chlorophenoxy)-3-(trifluoromethyl)-1-methyl-1H-Pyrazole-4-carbaldehyde-O-((2-chlo ropyridin-5-yl)methyl)oxime (9h). Yield 87\%, yellow solid, mp 60-62 ${ }^{\circ} \mathrm{C} .{ }^{1} \mathrm{H} \mathrm{NMR}(300 \mathrm{MHz}$, $\left.\mathrm{CDCl}_{3}\right): \delta 3.80\left(\mathrm{~s}, 3 \mathrm{H}, \mathrm{N}-\mathrm{CH}_{3}\right), 4.83\left(\mathrm{~s}, 2 \mathrm{H}, \mathrm{CH}_{2}\right), 6.58(\mathrm{~d}, J=7.8 \mathrm{~Hz}, 1 \mathrm{H}, \mathrm{Ar}-\mathrm{H}), 7.05-7.14(\mathrm{~m}$, 2H, Ar-H), 7.18 (d, $J=8.1 \mathrm{~Hz}, 1 \mathrm{H}, \mathrm{Py}-\mathrm{H}), 7.38-7.44$ (m, 2H, Py-H and Ar-H), 7.93 (s, 1H, $\mathrm{CH}=\mathrm{N}), 8.14(\mathrm{~d}, J=2.1 \mathrm{~Hz}, 1 \mathrm{H}, \mathrm{Py}-\mathrm{H}) .{ }^{13} \mathrm{C} \mathrm{NMR}\left(75 \mathrm{MHz}, \mathrm{CDCl}_{3}\right): \delta 35.3,72.6,100.5,115.1$, $118.9,122.5,122.8,123.9,124.8,127.8,130.9$, 132.0, 138.6, 138.7, 146.8, 149.2, 150.8, 151.2. Anal. Calcd for $\mathrm{C}_{18} \mathrm{H}_{13} \mathrm{Cl}_{2} \mathrm{~F}_{3} \mathrm{~N}_{4} \mathrm{O}_{2}$ : C, 48.56; H, 2.94; N, 12.58. Found: C, 48.65; H, 3.05; N, 12.42 .

5-(3-Chlorophenoxy)-3-(trifluoromethyl)-1-methyl-1H-Pyrazole-4-carbaldehyde-O-((2-chlo ropyridin-5-yl)methyl)oxime (9i). Yield 89\%, white solid, mp 65-67 ${ }^{\circ} \mathrm{C}$. ${ }^{1} \mathrm{H}$ NMR (300 MHz, $\left.\mathrm{CDCl}_{3}\right): \delta 3.75\left(\mathrm{~s}, 3 \mathrm{H}, \mathrm{N}-\mathrm{CH}_{3}\right), 4.82\left(\mathrm{~s}, 2 \mathrm{H}, \mathrm{CH}_{2}\right), 6.69(\mathrm{~d}, J=8.4 \mathrm{~Hz}, 1 \mathrm{H}, \mathrm{Ar}-\mathrm{H}), 6.83(\mathrm{~s}, 1 \mathrm{H}$, Ar-H), 7.10 (d, $J=7.5 \mathrm{~Hz}, 1 \mathrm{H}, \mathrm{Py}-\mathrm{H}), 7.19-7.23(\mathrm{~m}, 2 \mathrm{H}, \mathrm{Ar}-\mathrm{H}), 7.36\left(\mathrm{dd}, J_{1}=8.4 \mathrm{~Hz}, J_{2}=2.4 \mathrm{~Hz}\right.$, $1 \mathrm{H}, \mathrm{Py}-\mathrm{H}), 7.94(\mathrm{~s}, 1 \mathrm{H}, \mathrm{CH}=\mathrm{N}), 8.14(\mathrm{~d}, J=2.1 \mathrm{~Hz}, 1 \mathrm{H}, \mathrm{Py}-\mathrm{H}) .{ }^{13} \mathrm{C}$ NMR $\left(75 \mathrm{MHz}, \mathrm{CDCl}_{3}\right): \delta$ $35.3,72.6,100.8,113.4,115.9,118.9,122.4,123.9,124.3,130.7,131.9,135.4,138.6,138.7$, 146.5, 149.3, 150.9, 156.1. Anal. Calcd for $\mathrm{C}_{18} \mathrm{H}_{13} \mathrm{Cl}_{2} \mathrm{~F}_{3} \mathrm{~N}_{4} \mathrm{O}_{2}: \mathrm{C}, 48.56 ; \mathrm{H}, 2.94 ; \mathrm{N}, 12.58$. Found: C, 48.48; H, 2.95; N, 12.62 .

5-(4-Chlorophenoxy)-3-(trifluoromethyl)-1-methyl-1H-Pyrazole-4-carbaldehyde-O-((2-chlo ropyridin-5-yl)methyl)oxime (9j).Yield 92\%, yellow solid, mp99-101 ${ }^{\circ} \mathrm{C} .{ }^{1} \mathrm{H}$ NMR $(300 \mathrm{MHz}$, $\left.\mathrm{CDCl}_{3}\right): \delta 3.75\left(\mathrm{~s}, 3 \mathrm{H}, \mathrm{N}-\mathrm{CH}_{3}\right), 4.83\left(\mathrm{~s}, 2 \mathrm{H}, \mathrm{CH}_{2}\right), 6.77$ (d, J=8.7 Hz, 2H, Ar-H), $7.22(\mathrm{~d}, J=8.1$ $\mathrm{Hz}, 1 \mathrm{H}, \mathrm{Py}-\mathrm{H}), 7.27$ (d, J=8.7 Hz, 2H, Ar-H), 7.37 (dd, $\left.J_{1}=8.1 \mathrm{~Hz}, J_{2}=2.1 \mathrm{~Hz}, 1 \mathrm{H}, \mathrm{Py}-\mathrm{H}\right), 7.93$ $(\mathrm{s}, 1 \mathrm{H}, \mathrm{CH}=\mathrm{N}), 8.19(\mathrm{~d}, J=2.1 \mathrm{~Hz}, 1 \mathrm{H}, \mathrm{Py}-\mathrm{H}) .{ }^{13} \mathrm{C} \mathrm{NMR}\left(75 \mathrm{MHz}, \mathrm{CDCl}_{3}\right): \delta 35.3,72.6,100.7$, 116.5, 118.6, 122.4, 123.9, 129.2, 129.9, 131.9, 138.6, 138.8, 146.8, 149.4, 151.0, 154.2. Anal. Calcd for $\mathrm{C}_{18} \mathrm{H}_{13} \mathrm{Cl}_{2} \mathrm{~F}_{3} \mathrm{~N}_{4} \mathrm{O}_{2}$ : C, 48.56; H, 2.94; N, 12.58. Found: C, 48.62; H, 2.88; N, 12.71 .

5-(4-Fluorophenoxy)-3-(trifluoromethyl)-1-methyl-1H-Pyrazole-4-carbaldehyde-O-((2-chlo ropyridin-5-yl)methyl)oxime (9k). Yield 93\%, yellow solid, mp100-102 ${ }^{\circ} \mathrm{C}$. ${ }^{1} \mathrm{H}$ NMR (300 $\left.\mathrm{MHz}^{\mathrm{CDCl}}{ }_{3}\right): \delta 3.75\left(\mathrm{~s}, 3 \mathrm{H}, \mathrm{N}-\mathrm{CH}_{3}\right), 4.84\left(\mathrm{~s}, 2 \mathrm{H}, \mathrm{CH}_{2}\right), 6.77-6.81(\mathrm{~m}, 2 \mathrm{H}, \mathrm{Ar}-\mathrm{H}), 7.01(\mathrm{t}, J=8.7$ 
$\mathrm{Hz}, 2 \mathrm{H}, \mathrm{Ar}-\mathrm{H}), 7.22$ (d, $J=8.1 \mathrm{~Hz}, 1 \mathrm{H}, \mathrm{Py}-\mathrm{H}), 7.41$ (dd, $\left.J_{1}=8.1 \mathrm{~Hz}, J_{2}=2.1 \mathrm{~Hz}, 1 \mathrm{H}, \mathrm{Py}-\mathrm{H}\right), 7.93$ $(\mathrm{s}, 1 \mathrm{H}, \mathrm{CH}=\mathrm{N}), 8.18(\mathrm{~d}, J=2.1 \mathrm{~Hz}, 1 \mathrm{H}, \mathrm{Py}-\mathrm{H}) .{ }^{13} \mathrm{C} \mathrm{NMR}\left(75 \mathrm{MHz}, \mathrm{CDCl}_{3}\right): \delta 35.2,72.6,100.5$, 116.3, 116.4, 116.5, 116.7, 118.9, 122.4, 123.9, 131.9, 138.8, 147.3, 149.4, 150.9, 151.7, 157.3, 160.5. Anal. Calcd for $\mathrm{C}_{18} \mathrm{H}_{13} \mathrm{ClF}_{4} \mathrm{~N}_{4} \mathrm{O}_{2}: \mathrm{C}, 50.42 ; \mathrm{H}, 3.06 ; \mathrm{N}, 13.07$. Found: $\mathrm{C}, 50.48 ; \mathrm{H}, 3.15 ; \mathrm{N}$, 12.93 .

5-(4-Bromophenoxy)-3-(trifluoromethyl)-1-methyl-1H-Pyrazole-4-carbaldehyde-O-((2-chlo ropyridin-5-yl)methyl)oxime (9l).Yield 91\%, white solid, mp $106-108{ }^{\circ} \mathrm{C} .{ }^{1} \mathrm{H} \mathrm{NMR}(300 \mathrm{MHz}$, $\left.\mathrm{CDCl}_{3}\right): \delta 3.75\left(\mathrm{~s}, 3 \mathrm{H}, \mathrm{N}-\mathrm{CH}_{3}\right), 4.83\left(\mathrm{~s}, 2 \mathrm{H}, \mathrm{CH}_{2}\right), 6.72(\mathrm{~d}, J=9.0 \mathrm{~Hz}, 2 \mathrm{H}, \mathrm{Ar}-\mathrm{H}), 7.23(\mathrm{~d}, J=8.4$ $\mathrm{Hz}, 1 \mathrm{H}, \mathrm{Py}-\mathrm{H}), 7.36$ (dd, $\left.J_{1}=8.1 \mathrm{~Hz}, J_{2}=2.4 \mathrm{~Hz}, 1 \mathrm{H}, \mathrm{Py}-\mathrm{H}\right), 7.42$ (d, J=9.0 Hz, 2H, Ar-H), 7.93 $(\mathrm{s}, 1 \mathrm{H}, \mathrm{CH}=\mathrm{N}), 8.19(\mathrm{~d}, J=2.1 \mathrm{~Hz}, 1 \mathrm{H}, \mathrm{Py}-\mathrm{H}) .{ }^{13} \mathrm{C} \mathrm{NMR}\left(75 \mathrm{MHz}, \mathrm{CDCl}_{3}\right): \delta 35.3,72.6,100.7$, 116.5, 117.0, 118.9, 122.4, 123.9, 126.0, 131.9, 132.8, 138.7, 146.7, 149.4, 150.9, 154.8. Anal. Calcd for $\mathrm{C}_{18} \mathrm{H}_{13} \mathrm{BrClF}_{3} \mathrm{~N}_{4} \mathrm{O}_{2}$ : C, 44.15; H, 2.68; N, 11.44. Found: C, 44.10; H, 2.81; N, 11.36 .

5-(2,4-Dichlorophenoxy)-3-(trifluoromethyl)-1-methyl-1H-Pyrazole-4-carbalde-hyde-O-((2chloropyridin-5-yl)methyl)oxime (9m). Yield 89\%, yellow solid, mp 85-87 ${ }^{\circ} \mathrm{C} .{ }^{1} \mathrm{H}$ NMR $(300$ $\left.\mathrm{MHz}, \mathrm{CDCl}_{3}\right): \delta 3.80\left(\mathrm{~s}, 3 \mathrm{H}, \mathrm{N}-\mathrm{CH}_{3}\right), 4.84\left(\mathrm{~s}, 2 \mathrm{H}, \mathrm{CH}_{2}\right), 6.53(\mathrm{~d}, J=9.0 \mathrm{~Hz}, 1 \mathrm{H}, \mathrm{Ar}-\mathrm{H}), 7.11$ (dd, $\left.J_{1}=9.0 \mathrm{~Hz}, J_{2}=2.4 \mathrm{~Hz}, 1 \mathrm{H}, \mathrm{Ar}-\mathrm{H}\right), 7.23(\mathrm{~d}, J=8.1 \mathrm{~Hz}, 1 \mathrm{H}, \mathrm{Py}-\mathrm{H}), 7.39-7.43(\mathrm{~m}, 2 \mathrm{H}, \mathrm{Py}-\mathrm{H}$ and Ar-H), $7.95(\mathrm{~s}, 1 \mathrm{H}, \mathrm{CH}=\mathrm{N}), 8.19(\mathrm{~d}, J=2.1 \mathrm{~Hz}, 1 \mathrm{H}, \mathrm{Py}-\mathrm{H}) .{ }^{13} \mathrm{C} \mathrm{NMR}\left(75 \mathrm{MHz}, \mathrm{CDCl}_{3}\right): \delta 35.3$, 72.7, 100.5, 115.9, 118.8, 122.4, 123.8, 123.9, 127.8, 129.6, 130.6, 131.9, 138.4, 138.6, 146.4, 149.2, 149.9, 151.0. Anal. Calcd for $\mathrm{C}_{18} \mathrm{H}_{12} \mathrm{Cl}_{3} \mathrm{~F}_{3} \mathrm{~N}_{4} \mathrm{O}_{2}$ : C, 45.07; H, 2.52; N, 11.68. Found: C, 45.19; H, 2.61; N, 11.53 .

5-(3-Nitrophenoxy)-3-(trifluoromethyl)-1-methyl-1H-Pyrazole-4-carbaldehyde-O-((2-chlor opyridin-5-yl)methyl)oxime (9n). Yield 87\%, yellow solid, mp 81-83 ${ }^{\circ} \mathrm{C} .{ }^{1} \mathrm{H}$ NMR (300 MHz, $\left.\mathrm{CDCl}_{3}\right): \delta 3.80\left(\mathrm{~s}, 3 \mathrm{H}, \mathrm{N}-\mathrm{CH}_{3}\right), 4.80\left(\mathrm{~s}, 2 \mathrm{H}, \mathrm{CH}_{2}\right), 7.13-7.20\left(\mathrm{~m}, 2 \mathrm{H}, \mathrm{Py}-\mathrm{H}\right.$ and Ar-H), $7.38\left(\mathrm{dd}, J_{1}\right.$ $\left.=8.4 \mathrm{~Hz}, J_{2}=2.4 \mathrm{~Hz}, 1 \mathrm{H}, \mathrm{Py}-\mathrm{H}\right), 7.45-7.67(\mathrm{~m}, 2 \mathrm{H}, \mathrm{Ar}-\mathrm{H}), 7.96-7.99(\mathrm{~m}, 2 \mathrm{H}, \mathrm{CH}=\mathrm{N}$ and Ar-H), $8.05(\mathrm{~d}, J=2.1 \mathrm{~Hz}, 1 \mathrm{H}, \mathrm{Py}-\mathrm{H}) .{ }^{13} \mathrm{C} \mathrm{NMR}\left(75 \mathrm{MHz}, \mathrm{CDCl}_{3}\right): \delta 35.4,72.5,100.9,110.6,116.6$, $118.9,121.2$, 122.4, 123.9, 130.7, 131.8, 138.2, 138.9, 145.9, 148.9, 149.1, 150.9, 155.8. Anal. Calcd for $\mathrm{C}_{18} \mathrm{H}_{13} \mathrm{ClF}_{3} \mathrm{~N}_{5} \mathrm{O}_{4}$ : C, 47.43; H, 2.87; N, 15.37. Found: C, 47.36; H, 2.81; N, 15.42 .

5-(3-Methylphenylthio)-3-(trifluoromethyl)-1-methyl-1H-Pyrazole-4-carbaldehyde-O-((2chloropyridin-5-yl)methyl)oxime (12a). Yield 86\%, white solid, mp 38-40 ${ }^{\circ} \mathrm{C} .{ }^{1} \mathrm{H}$ NMR $(300$ $\left.\mathrm{MHz}, \mathrm{CDCl}_{3}\right): \delta 2.29\left(\mathrm{~s}, 3 \mathrm{H}, \mathrm{Ar}-\mathrm{CH}_{3}\right), 3.87\left(\mathrm{~s}, 3 \mathrm{H}, \mathrm{N}-\mathrm{CH}_{3}\right), 5.09\left(\mathrm{~s}, 2 \mathrm{H}, \mathrm{CH}_{2}\right), 6.79(\mathrm{~d}, J=7.8 \mathrm{~Hz}$, 1H, Ar-H), 6.86 (s, 1H, Ar-H), 7.04 (d, J=7.5 Hz, 1H, Ar-H), 7.16 (t, J=7.5 Hz, 1H, Ar-H), 7.25 $(\mathrm{d}, J=8.1 \mathrm{~Hz}, 1 \mathrm{H}, \mathrm{Py}-\mathrm{H}), 7.65$ (dd, $\left.J_{1}=8.4 \mathrm{~Hz}, J_{2}=2.1 \mathrm{~Hz}, 1 \mathrm{H}, \mathrm{Py}-\mathrm{H}\right), 8.15(\mathrm{~s}, 1 \mathrm{H}, \mathrm{CH}=\mathrm{N}), 8.37$ $(\mathrm{d}, J=2.1 \mathrm{~Hz}, 1 \mathrm{H}, \mathrm{Py}-\mathrm{H}) .{ }^{13} \mathrm{C} \mathrm{NMR}\left(75 \mathrm{MHz}, \mathrm{CDCl}_{3}\right): \delta 21.3,37.8,72.9,117.5,119.0,122.6$, 123.9, 124.7, 128.1, 128.2, 129.5,131.9, 132.7, 133.9, 139.4, 139.8, 140.2, 150.0, 151.1. Anal. Calcd for $\mathrm{C}_{19} \mathrm{H}_{16} \mathrm{ClF}_{3} \mathrm{~N}_{4} \mathrm{OS}$ : C, 51.76; H, 3.66; N, 12.71. Found: C, 51.68; H, 3.78; N, 12.58 .

5-(4-Chlorophenylthio)-3-(trifluoromethyl)-1-methyl-1H-Pyrazole-4-carbaldehyde-O-((2chloropyridin-5-yl)methyl)oxime (12b). Yield 93\%, white solid, mp 79-81 ${ }^{\circ} \mathrm{C}$. ${ }^{1} \mathrm{H}$ NMR (300 $\left.\mathrm{MHz}, \mathrm{CDCl}_{3}\right): \delta 3.89\left(\mathrm{~s}, 3 \mathrm{H}, \mathrm{N}-\mathrm{CH}_{3}\right), 5.08\left(\mathrm{~s}, 2 \mathrm{H}, \mathrm{CH}_{2}\right), 6.95(\mathrm{~d}, J=8.7 \mathrm{~Hz}, 2 \mathrm{H}, \mathrm{Ar}-\mathrm{H}), 7.24(\mathrm{~d}, J$ $=8.4 \mathrm{~Hz}, 1 \mathrm{H}, \mathrm{Py}-\mathrm{H}), 7.27(\mathrm{~d}, J=8.4 \mathrm{~Hz}, 2 \mathrm{H}, \mathrm{Ar}-\mathrm{H}), 7.62\left(\mathrm{dd}, J_{1}=8.1 \mathrm{~Hz}, J_{2}=2.4 \mathrm{~Hz}, 1 \mathrm{H}, \mathrm{Py}-\mathrm{H}\right)$, $8.14(\mathrm{~s}, 1 \mathrm{H}, \mathrm{CH}=\mathrm{N}), 8.36(\mathrm{~d}, J=2.1 \mathrm{~Hz}, 1 \mathrm{H}, \mathrm{Py}-\mathrm{H}) .{ }^{13} \mathrm{C} \mathrm{NMR}\left(75 \mathrm{MHz}, \mathrm{CDCl}_{3}\right): \delta 37.8,72.9$, 
117.7, 118.9, 122.5, 123.9, 128.8, 129.8, 131.6, 131.9, 132.9, 133.4, 139.3, 139.9, 149.9, 151.1 . Anal. Calcd for $\mathrm{C}_{18} \mathrm{H}_{13} \mathrm{Cl}_{2} \mathrm{~F}_{3} \mathrm{~N}_{4} \mathrm{OS}$ : C, 46.87; H, 2.84; N, 12.15. Found: C, 46.93; H, 2.98; N, 12.09 .

5-(t-Butylthio)-3-(trifluoromethyl)-1-methyl-1H-Pyrazole-4-carbaldehyde-O-((2-chloropyri din-5-yl)methyl)oxime (12c). Yield 88\%, white solid, mp 97-99 ${ }^{\circ} \mathrm{C}$.

${ }^{1} \mathrm{H}$ NMR (300 MHz, $\left.\mathrm{CDCl}_{3}\right): \delta 1.29\left(\mathrm{~s}, 9 \mathrm{H}, \mathrm{C}\left(\mathrm{CH}_{3}\right)_{3}\right), 4.01\left(\mathrm{~s}, 3 \mathrm{H}, \mathrm{N}-\mathrm{CH}_{3}\right), 5.14\left(\mathrm{~s}, 2 \mathrm{H}, \mathrm{CH}_{2}\right)$, $7.31(\mathrm{~d}, J=8.4 \mathrm{~Hz}, 1 \mathrm{H}, \mathrm{Py}-\mathrm{H}), 7.72\left(\mathrm{dd}, J_{1}=8.1 \mathrm{~Hz}, J_{2}=2.4 \mathrm{~Hz}, 1 \mathrm{H}\right.$, Py-H), $8.12(\mathrm{~s}, 1 \mathrm{H}, \mathrm{CH}=\mathrm{N})$, $8.42(\mathrm{~d}, J=2.1 \mathrm{~Hz}, 1 \mathrm{H}, \mathrm{Py}-\mathrm{H}) .{ }^{13} \mathrm{C}$ NMR $\left(75 \mathrm{MHz}, \mathrm{CDCl}_{3}\right): \delta 31.1,37.7,51.6,72.9,118.4,119.0$, 122.6, 123.9, 132.1, 136.2, 139.5, 141.7, 149.9, 151.1. Anal. Calcd for $\mathrm{C}_{16} \mathrm{H}_{18} \mathrm{ClF}_{3} \mathrm{~N}_{4} \mathrm{OS}$ : C, 47.23; H, 4.46; N, 13.77. Found: C, 47.35; H, 4.38; N, 13.80 .

5-(Methylthio)-3-(trifluoromethyl)-1-methyl-1H-Pyrazole-4-carbaldehyde-O-((2-chloropyri din-5-yl)methyl)oxime (12d). Yield 91\%, white solid, mp 73-75 ${ }^{\circ} \mathrm{C}$.

${ }^{1} \mathrm{H}$ NMR $\left(300 \mathrm{MHz}, \mathrm{CDCl}_{3}\right): \delta 2.30\left(\mathrm{~s}, 3 \mathrm{H}, \mathrm{CH}_{3}\right), 4.01\left(\mathrm{~s}, 3 \mathrm{H}, \mathrm{N}-\mathrm{CH}_{3}\right), 5.17\left(\mathrm{~s}, 2 \mathrm{H}, \mathrm{CH}_{2}\right), 7.33(\mathrm{~d}$, $J=8.1 \mathrm{~Hz}, 1 \mathrm{H}, \mathrm{Py}-\mathrm{H}), 7.74\left(\mathrm{dd}, J_{1}=8.4 \mathrm{~Hz}, J_{2}=2.4 \mathrm{~Hz}, 1 \mathrm{H}, \mathrm{Py}-\mathrm{H}\right), 8.17(\mathrm{~s}, 1 \mathrm{H}, \mathrm{CH}=\mathrm{N}), 8.44(\mathrm{~d}$, $J=1.8 \mathrm{~Hz}, 1 \mathrm{H}, \mathrm{Py}-\mathrm{H}) .{ }^{13} \mathrm{C} \mathrm{NMR}\left(75 \mathrm{MHz}, \mathrm{CDCl}_{3}\right): \delta 18.9,37.5,72.9,116.0,119.1,122.6,124.0$, 132.1, 137.5, 139.3, 140.6, 149.9, 151.1. Anal. Calcd for $\mathrm{C}_{13} \mathrm{H}_{12} \mathrm{ClF}_{3} \mathrm{~N}_{4} \mathrm{OS}: \mathrm{C}, 42.80 ; \mathrm{H}, 3.32 ; \mathrm{N}$, 15.36. Found: C, $42.65 ; \mathrm{H}, 3.38 ; \mathrm{N}, 15.45$.

\section{Biological assays}

Bioassay of insecticidal activities. The insects, pea aphids (Aphis craccivora), were reared in the laboratory. The insecticidal activities of compounds 9a-9n, 12a-12d against pea aphids (Aphis craccivora) were tested according to a reported procedure. ${ }^{22}$ The insects were placed in a room maintained at $26{ }^{\circ} \mathrm{C}, 60 \%$ relative humidity, and a $14 \mathrm{~h}$ photoperiod. Stock solutions of each test sample was prepared in acetone at a concentration of $0.5 \mathrm{mg} / \mathrm{mL}$, and then diluted to the required concentration with water containing TW-20. Tender shoots of soybean with 60 insects were dipped in the diluted solutions of the chemicals for $5 \mathrm{~s}$, then the superfluous liquor was removed, and they were kept in the conditioned room for normal cultivation. The mortality was evaluated by the number or size of live larvae in the treated bottles relative to that in the untreated controls after $24 \mathrm{~h}$ and $48 \mathrm{~h}$, respectively. Controls were performed under the same conditions. Each treatment was performed three times. The data were subjected to probit analysis.

Bioassay of plant growth regulatory activities. The plant growth regulatory activities of compounds 9a-9n, 12a-12d were evaluated by means of cucumber cotyledon test according to a reported procedure. ${ }^{23}$ The cucumber seeds (JINKE, No.4) were supplied by the Biological Assay Center, Nankai University, China. These seeds were incubated at $24^{\circ} \mathrm{C}$ in a darkroom for 3 days, and 10 pieces of cotyledons of the same size were selected. The test samples were dissolved in DMF at a concentration of $10 \mu \mathrm{g} / \mathrm{mL}$. A sample solution $(0.3 \mathrm{~mL})$ was sprayed over a filter paper ( $6 \mathrm{~cm}$ diameter), and solvent was volatilized to dryness on air. The filter paper thus prepared was placed into an incubation vessel $(6 \mathrm{~cm}$ diameter) and soaked with distilled water $(3 \mathrm{~mL})$. Finally, 10 pieces of cotyledons were added. These cotyledons were incubated at $24{ }^{\circ} \mathrm{C}$ in a darkroom for 3 days. The rhizogenesis numbers of every 10 pieces of hypocotyls were measured. In contrast, 
the distilled water was used as a control. Each test was performed in triplicate.

Statistical Analysis. The results were expressed as means \pm standard deviation (SD) of three parallel experiments. Data were analyzed by Student's $t$-test. The LC $_{50}$ (median lethal concentration) was analyzed using probit analysis performed with the statistical software SAS.

\section{Acknowledgement}

This work was financially supported by the National Natural Science Foundation of China (No. 20772068) and the National Key Project of Scientific and Technical Supporting Programs of China (2006BAE01A01-5).

\section{References and Notes}

1. (a) Haaf, K.; Kehne, H.; Bauer, K.; Bieringer, H. Ger.Offen DE 4122905, 1993; Chem. Abstr. 1993, 119, 72496. (b) Finkelstein, B. L.; Martz, M. A.; Strock, C. J. Pestic. Sci. 1997, 50, 319. (c) Zhang, A. G.; Kayser, H.; Maienfisch, P.; Casida, J. E. J. Neurochem. 2000, 75, 1294. (d) Ballistreri, F. P.; Barresi, V.; Consiglio, G.; Fortuna, C. G.; Longo, M. L.; Musumarra, G. Arkivoc 2003, (i), 105. (e) Liu, J. B.; Li, L. C.; Dai, H.; Liu, Z.; Fang, J. X. J. Organomet. Chem. 2006, 691, 2686.

2. (a) Jo, Y. W.; Im, W. B.; Rhee, J. K.; Shim, M. J.; Kim, W. B.; Choi, E. C. Bioorg. Med. Chem. 2004, 12, 5909. (b) Singh, P.; Kaur, P.; Luxami, V.; Kaur, S.; Kumar, S. Bioorg. Med. Chem. 2007, 15, 2386.

3. Kagabu, S.; Ishihara, R.; Hieda, Y.; Nishimura, K.; Naruse, Y. J. Agric. Food Chem. 2007, 55, 812.

4. (a) Liu, Z. W.; Han, Z. J.; Wang, Y. C.; Zhang, L. C.; Zhang, H. W.; Liu, C. J. Pest Manage. Sci. 2003, 59, 1355. (b) Nauen, R.; Denholm, I. Arch. Insect Biochem. Physiol. 2005, 58, 200.

5. (a) Tian, Z. Z.; Shao, X. S.; Li, Z.; Qian, X. H.; Huang, Q. C. J. Agric. Food Chem. 2007, 55, 2288. (b) Shao, X. S.; Zhang, W. W.; Peng, Y. Q.; Li, Z.; Tian, Z. Z.; Qian, X. H. Bioorg. Med. Chem. Lett. 2008, 18, 6513.

6. (a) Daidone, G.; Maggio, B.; Plescia, S.; Raff, D.; Musiu, C.; Milia, C.; Perra, G.; Marongiu, M. E. Eur. J. Med. Chem. 1998, 33, 375. (b) Singh, N.; Sangwan, N. K.; Dhindsa, K. S. Pest Manage. Sci. 2000, 56, 284. (c) Daidone, G.; Raffa, D.; Plescia, F.; Maggio, B.; Roccaro, A. Arkivoc 2002, (xi), 227; (d) Migliara, O.; Plescia, S.; Diana, P.; Di Stefano, V.; Camarda, L.; Dall'Olio, R. Arkivoc 2004, (v), 44; (e) Katritzky, A. R.; Vakulenko, A. V.; Akue-Gedu, R.; Gromova, A. V.; Witek, R.; Rogers, J. W. Arkivoc 2007, (i), 9.

7. Li, Y.; Zhang, H. Q.; Liu, J.; Yang, X. P.; Liu, Z. J. J. Agric. Food Chem. 2006, 54, 3636. 
8. (a) Sammelson, R. E.; Caboni, P.; Durkin, K. A.; Casida, J. E. Bioorg. Med. Chem. 2004, 12, 3345. (b) Chen, L.; Ou, X. M.; Mao, C. H.; Shang, J.; Huang, R. Q.; Bi, F. C.; Wang, Q. M. Bioorg. Med. Chem. 2007, 15, 3678.

9. Meazza, G.; Bettarini, F.; La Porta, P.; Piccardi, P.; Signorini, E.; Portoso, D.; Fornara, L. Pest Manage. Sci. 2004, 60, 1178.

10. Gamage, S. A.; Spicer, J. A.; Rewcastle, G. W.; Milton, J.; Sohal, S.; Dangerfield, W.; Mistry, P.; Vicker, N.; Charlton, P. A.; Denny, W. A. J. Med. Chem. 2002, 45, 740.

11. Caboni, P.; Sammelson, R. E.; Casida, J. E. J. Agric. Food Chem. 2003, 51, 7055.

12. (a) Rossello, A.; Bertini, S.; Lapucci, A.; Macchia, M.; Martinelli, A.; Rapposelli, S.; Herreros, E.; Macchia, B. J. Med. Chem. 2002, 45, 4903. (b) Park, H. J.; Lee, K.; Park, S. J.; Ahn, B.; Lee, J. C.; Cho, H. Y.; Lee, K. I. Bioorg. Med. Chem. Lett. 2005, 15, 3307. (c) Hofmann, M.; Langewald, J.; Kuhn, D. G.; Oloumi-Sadeghi, H.; Braun, F. J.; Culbertson, D. L. PCT Int. Appl. WO 2006125637, 2006; Chem. Abstr. 2006, 145, 501089. (d) Ramalingan, C.; Park, Y. T.; Kabilan, S. Eur. J. Med. Chem. 2006, 41, 683.

13. Sun, R. F.; Lü, M. Y.; Chen, L.; Li, Q. S.; Song, H. B.; Bi, F. C.; Huang, R. Q.; Wang, Q. M. J. Agric. Food Chem. 2008, 56, 11376.

14. (a) Smart, B. E. J. Fluorine Chem. 2001, 109, 3. (b) Gong, Y. F.; Kato, K. Curr. Org. Chem. 2004, 8, 1659. (c) Begue, J. P.; Bonnet-Delpon, D.; Crousse, B.; Legros, J. Chem. Soc. Rev. 2005, 34, 562. (d) Huang, W.; Yang, G. F. Bioorg. Med. Chem. 2006, 14, 8280.

15. Lantzsch, R. Eur. Pat. Appl. EP 0546418, 1993; Chem. Abstr. 1993, 119, 160133.

16. Gao, R. J.; Bai, J. J.; Liu, X. L.; Li, J. Y. Chin. J. Pestic. 1997, 36, 13.

17. Lee, L. F.; Schleppnik, F. M.; Schneider, R. W.; Campbell, D. H. J. Heterocycl. Chem. 1990, $27,243$.

18. Gilbert, A. M.; Bursavich, M. G.; Lombardi, S.; Georgiadis, K. E.; Reifenberg, E.; Flannery, C. R.; Morris, E. A. Bioorg. Med. Chem. Lett. 2007, 17, 1189.

19. (a) Takaishi, H.; Hamaguchi, H.; Nishimura, A.; Yanaka, K. JP. Pat. 62053969, 1987; Chem. Abstr. 1987, 107, 115585; (b) Liu, X. H.; Cui, P.; Song, B. A.; Bhadury, P. S.; Zhu, H. L.; Wang, S. F. Bioorg. Med. Chem. 2008, 16, 4075.

20. Colorless blocks of $12 \mathbf{b}(0.16 \mathrm{~mm} \times 0.14 \mathrm{~mm} \times 0.12 \mathrm{~mm})$ were counted on a quartz fiber withprotection oil. Cell dimensions and intensities were measured at $113 \mathrm{~K}$ on a Rigaku Saturn 70 CCD area detector diffractometer with confocal monochromated Mo K $\alpha$ radiation $(\lambda=0.71073 \AA)$. Crystallographic data (excluding structure factors) of $\mathbf{1 2 b}$ have been deposited at Cambridge Crystallographic Data Centre as supplementary publication No. CCDC 702059. Copies of the data can be obtained, free of charge, on application to CCDC, 12 Union Road, Cambridge CB2 1EZ, UK (fax: +44 (0)1223 336033 or e-mail: deposit@ccdc.cam.ac.uk).

21. Harada, J.; Harakawa, M.; Ogawa, K. Acta Cryst. 2004, B60, 578.

22. Tian, Z. Z.; Jiang, Z. X.; Li, Z.; Song, G. H.; Huang, Q. C. J. Agric. Food Chem. 2007, 55, 143.

23. Jin, Z.; Hu, Y.; Huo, A. H.; Tao, W. F.; Shao, L.; Liu, J. B.; Fang, J. X. J. Organomet. Chem. 
2006, 691, 2340. 